Tôhoku Math. Journ.

30 (1978), 373-422.

\title{
SEMI-SIMPLICIAL WEIL ALGEBRAS AND CHARACTERISTIC CLASSES
}

\author{
Franz W. Kamber* and PhilipPe Tondeur*
}

(Received January 5, 1977)

\begin{abstract}
The purpose of this paper is the construction and cohomological study of semi-simplicial models for the Weil algebra of a Lie algebra. The geometric context where the authors introduced these algebras is the construction of generalized characteristic classes for foliated bundles. There are two main aspects to the results of this paper. The first is the homological equivalence of all semi-simplicial Weil algebras even after passing to basic elements with respect to a subalgebra and to quotients by certain characteristic filtration ideals. The geometric consequence is that the generalized characteristic homomorphisms defined on these various complexes all have the same domain of universal generalized characteristic invariants. The second aspect is a comparison map from the ordinary Weil algebra to the semi-simplicial Weil algebra realizing a homology isomorphism after passing to basic elements with respect to a subalgebra and to quotients by characteristic filtration ideals. The geometric consequence is a comparison of the characteristic class constructions on the various complexes considered, which is also of significance for explicit computations.
\end{abstract}

\section{Contents}

1. Introduction $\ldots \ldots \ldots \ldots \ldots \ldots \ldots \ldots \ldots \ldots \ldots \ldots \ldots \ldots \ldots \ldots \ldots \ldots$

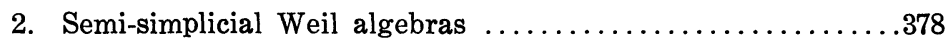

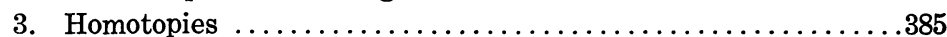

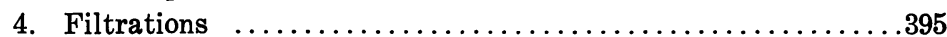

5. Isomorphism theorems........................408

6. Generalized characteristic homomorphism $\ldots \ldots \ldots \ldots \ldots . \ldots 414$

1. Introduction. The purpose of this paper is the construction and cohomological study of semi-simplicial models for the Weil algebra of a Lie algebra as announced in the notes [13] and used in our subsequent work [14], [15].

To appreciate the results of this paper it will be useful to recall the geometric context leading to the algebraic constructions of this paper. This context is the construction of characteristic classes for

* This work was partially supported by a grant from the National Science Foundation. AMS 1972 subject classification: Primary 5732, Secondary 5736.

Key words: Characteristic classes, foliations, foliated bundles, Weil algebra, semi-simplicial Weil algebras. 
foliated bundles. Since an account of this subject has appeared (beside the original papers) in the form of detailed notes of our lectures [16], we will be brief and refer to these notes for more details.

A foliated $G$-bundle is a principal $G$-bundle $P \stackrel{\pi}{\rightarrow} M$ equipped with (i) a foliation (involutive subbundle) $L \subset T_{M}$ of codimension $q$ on $M$, (ii) a foliation $\widetilde{L} \subset T_{P}$ on $P$ which is $G$-equivariant, projects onto $L$ under $\pi$, and such that $\widetilde{L}_{u} \cap G_{u}=\{0\}$ for every $u \in P$ and $G_{u}$ the tangent space to the fiber through $u$. An adapted connection is a connection $\omega$ on $P$ such that $\omega_{u}\left(\widetilde{L}_{u}\right)=0$ for each $u \in P$, i.e., the horizontal subspace of the connection contains the subspace $\widetilde{L}_{u}$ for each $u \in P$. Let $\Omega^{*}(P)$ denote the De Rham complex of global forms on $P$. The Weil homomorphism

$$
k(\omega): W^{*}(\mathrm{~g}) \rightarrow \Omega^{*}(P)
$$

of an adapted connection has certain characteristic filtration preserving properties, which leads directly to the construction of a generalized characteristic homomorphism (see [16], Chapter 4). This homomorphism is defined on the cohomology of the truncated Weil algebra $W(\mathfrak{g})_{q}$ or more generally the relative truncated Weil algebra $W(\mathfrak{g}, H)_{q}$. The closed subgroup $H \subset G$ reflects the appearance of an additional $H$-reduction of the $G$-bundle $P$. The characteristic homomorphism measures the incompatibility of this geometric structure with the foliated bundle structure of $P$.

One obstacle to the application of this construction to holomorphic and algebraic bundles is the fact that no global connections need then exist. But what one can use instead is a family of local connections on the bundle $P$ restricted to the sets of a cohomologically trivial open covering $\mathscr{C}$ of $M$. Even in the smooth case connections are often given in this way by local data, and a direct construction of the characteristic homomorphism of $P$ via these data is desirable, regardless of the fact that they can be constructed by the use of a global connection in $P$. This leads automatically to semi-simplicial methods, since the resulting invariants are defined in Čech cohomology. The need for semi-simplicial methods arises also in the construction of characteristic classes for simplicial bundles, where the characteristic homomorphism takes values in the simplicial De Rham cohomology.

We explain the basic idea for the construction of the generalized characteristic homomorphism $\Delta(P)_{*}$ in this more general context (see [13] [14] [15]). Let $\mathscr{U}=\left(U_{j}\right)$ be an open covering of the base space $M$ of the foliated $G$-bundle $P \rightarrow M$, such that each $P / U_{j}$ admits a connection $\omega_{j}$ adapted to the foliation. For the family $\omega=\left(\omega_{j}\right)$ there is 
then a generalization of the Weil homomorphism (1.1) to a $g$-DG-algebra homomorphism

$$
k_{1}(\boldsymbol{\omega}): W_{1}^{*}(\mathrm{~g}) \rightarrow \breve{C}\left(\mathscr{\mathscr { C }}, \pi_{*} \Omega_{P}^{*}\right) .
$$

The domain is the semi-simplicial Weil algebra defined in 2.18 (the subscript hints to the Definition 2.22 of a whole sequence $W_{s}(\mathrm{~g}), s \geqq 0$ of such Weil algebras). The target is the $g$-DG-algebra of Cech cochains of $\mathscr{U}$ with coefficients in the direct image $\pi_{*} \Omega_{P}^{*}$ of the De Rham sheaf complex $\Omega_{P}^{*}$ of local forms on $P$. The map (1.2) is formally constructed as a map of cochain complexes. This semi-simplicial Weil homomorphism is filtration preserving for certain characteristic filtrations on domain and target. This leads to a semi-simplicial characteristic homomorphism, defined on the cohomology of the truncated semi-simplicial Weil algebra $W_{1}(\mathfrak{g})_{q}$, or more generally the relative truncated semi-simplicial Weil algebra $W_{1}(\mathfrak{g}, H)_{q}$. The subgroup $H \subset G$ again reflects the appearance of a geometric structure on $P$, whose incompatibility with the foliated bundle structure of $P$ is measured by this characteristic class construction.

The results of this paper are twofold. First the complexes $W(\mathfrak{g}, H)_{q}$, $W_{1}(\mathfrak{g}, H)_{q}$ and similarily constructed complexes $W_{s}(\mathfrak{g}, H)_{q}$ for $s \geqq 0$ are shown to be homologically equivalent. The geometric implication is that the generalized characteristic homomorphisms derived on these complexes from the maps $k(\omega), k_{1}(\omega)$ and similarily constructed Weil homomorphisms $k_{s}(\omega)$ all have the same domain of universal generalized characteristic invariants. Secondly a comparison map

$$
\lambda: W(\mathfrak{g}) \rightarrow W_{1}(\mathfrak{g})
$$

is constructed which realizes this homology isomorphism after passing in (1.3) to basic elements with respect to a subgroup, and to the quotients by the relevant filtration ideals. The geometric implication is the comparison of the constructions arising from (1.1) and (1.2), in the case when both apply, as e.g., in the situations where global adapted connections exist. This comparison map or difference map is not only significant for explicit computations, but is a technical tool which makes its appearance in practically all aspects of the theory of generalized characteristic classes. In its simplest form it is an algebraic equivalent for the Weil lemma establishing the homotopy between the characteristic homomorphisms corresponding to two connections. It is explained in [25] how the construction of characteristic classes for foliations by Bott-Shulman-Stasheff [4] in the context of a semi-simplicial De Rham 
theory for $B \Gamma$ relates to our construction via the semi-simplicial Weil algebra $W_{1}$.

The organization of the paper is as follows. Section 2 is devoted to the construction of a sequence of semi-simplicial Weil algebras $W_{s}$ for $s \geqq 0$, with $W_{0}=W$. The need for $W_{1}$ has been explained above (see also Section 6 of this paper). The need for an iteration of this construction arises e.g. in the proof of the independence of the semisimplicial characteristic homomorphism from the choice of a family of local adapted connections (Proposition 6.11). The crucial argument is contained in the commutative diagram 3.33. For a functorial construction of $W_{s}$ the appropriate domain is the category $\mathscr{L}_{8}$ of Lie algebras $L$ over a fixed Lie algebra g. Then $W_{s}(L)$ for $s \geqq 0$ is recursively defined as a cochain complex over the semi-simplicial point with coefficients in a local system of $\mathfrak{g}$-DG-algebras (see Definitions 2.18 and 2.22). This construction assigns to every Lie algebra $L \supset \mathfrak{g}$ a sequence $W_{\mathrm{s}}(L), s \geqq 0$ of $\mathrm{g}$-DG-algebras in a contravariant functorial manner. These DG-algebras are all acyclic (see 5.9). The cohomologically interesting objects $W_{s}(L, \mathfrak{h})_{q}$ arise from these algebras by passing to basic elements with respect to subalgebras $\mathfrak{h} \subset \mathfrak{g}$, and to quotient algebras by filtration ideals arising naturally out of geometric considerations (see Definitions 4.61 and 5.3). The main result of this paper is the fact that the homology $H\left(W_{s}(L, \mathfrak{h})_{q}\right)$ is the same for all $s \geqq 0$ and in fact also independent of the choice of the Lie algebra $L$ over $g$. It can therefore be evaluated on the particular algebra $L=\mathfrak{g}$. Thus the complexes $W_{s}(L, \mathfrak{h})_{q}$ for $s \geqq 0$ and $L$ over $\mathfrak{g}$ all realize the homology $H\left(W(\mathfrak{g}, \mathfrak{h})_{q}\right.$ ) (see Theorem 5.5 and Corollary 5.7 for precise statements, and Remark 5.22 for the change from Lie algebra pairs $(\mathfrak{g}, \mathfrak{h})$ to pairs $(G, H)$ of Lie groups). The main fact to establish is the independence of the homology $H\left(W_{0}(L, \mathfrak{h})_{q}\right)$ from $L$. This is done in Section 4 (Theorem 4.63 and Corollary 4.76). The remaining facts follow by general spectral sequence arguments which are carried out in Section 5. We expect that the general principle behind this construction will turn out to be useful in other contexts. The explicit computation of the algebras $H\left(W(\mathfrak{g}, \mathfrak{h})_{q}\right)$ is the subject of the note [12] (see also [16], Chapter 5 and the detailed treatment in [20]). It is not discussed in this paper.

In Section 3 we construct the map $\lambda$ of (1.3) and analyze its properties. Since $W_{1}$ is defined as a complex of cochains, we consider more generally a local system $E$ of $\mathfrak{g}$-DG-algebras on a semi-simplicial set $S$, equipped with a family $\omega=\left(\omega_{j}\right)$ of connections $\omega_{j}: \wedge \mathfrak{g}^{*} \rightarrow E_{j}$ for the 0 -simplices $j \in S_{0}$ of $S$. We construct then a map 


$$
\lambda_{E}: W(\mathfrak{g}) \rightarrow C(S, E)
$$

whose properties are summarized in Theorem 3.25. This map has a certain analogy to the maps defined by Bott in Section 5 of [1]. We draw particular attention to the commutation of this map with the differentials on both sides of (1.4). This fact is based on the simplicial Stokes formula of Lemma 3.5. Applied to $W_{1}$, it leads to property (i) for $\lambda$ in Theorem 3.26. For the map $\lambda^{1}: W \rightarrow W \otimes W$ in particular this proves that it is a homotopy between the universal connections $W \rightarrow W \otimes 1$ and $W \rightarrow 1 \otimes W$. Thus $\lambda$ can be viewed as a generalization of the classical Weil lemma on characteristic forms. More applications of $\lambda$ can be found in Chapter 5 of [16], and in [17] [18].

In Section 4 we discuss filtrations. In contrast to the canonical gfiltration $F(\mathfrak{g})$ defined on every $\mathfrak{g}$-DG-algebra (see Definition 4.1), we consider on the Weil algebra $W(L)$ for $L \in \mathscr{L}_{\mathrm{B}}$ another even filtration $F_{0}^{2 p}(\mathrm{~g}) W(L)$ by $\mathrm{g}$-DG-ideals. This filtration is defined in 4.30 and is functorial not only on $\mathscr{L}_{g}$, but also for linear maps $\varphi: L^{\prime} \rightarrow L$ over $g$ (see Corollary 4.32). The technical result in this direction is Proposition 4.7, which is a refined statement of the universal property of the Weil algebra. It is then shown that the universal maps $\lambda^{l}: W(L)^{\circ} \rightarrow W\left(L^{l+1}\right)^{\circ-l}$ preserve the filtration $F_{0}(\mathfrak{g})$. This statement 4.41 is vital, since it implies e.g., that homotopies between Weil homomorphisms for two connections pass to quotients by the filtration ideals $F_{0}^{2 p}(\mathfrak{g})$ (see Proposition 4.54). This property does notably not hold for the canonical filtrations on domain and target. The definition of the relative truncated Weil algebras $W_{0}(L, \mathfrak{h})_{q}$ and $W(\mathfrak{g}, \mathfrak{h})_{q}$ as the quotients by the filtration ideals $F_{0}^{2(q+1)}(\mathfrak{g}) W(L, \mathfrak{h})$ and $F^{2(q+1)}(\mathfrak{g}) W(\mathfrak{g}, \mathfrak{h})$ is given in 4.61 and 4.62. The main result of this section and indeed the crucial fact in this paper is the homotopy equivalence of the DG-algebras $W_{0}(L, \mathfrak{h})_{q}$ and $W(\mathfrak{g}, \mathfrak{h})_{q}$, which holds even functorially for linear maps $L^{\prime} \rightarrow L$ over $\mathrm{g}$. The precise statements are Theorem 4.63 and Corollary 4.67. We emphasize again that this is not true for the truncation with respect to the canonical $\mathfrak{g}$-filtration on $W(L, \mathfrak{h})$ (see Remark 4.69). A geometric use of this realization of $H\left(W(\mathfrak{g}, \mathfrak{h})_{q}\right)$ by the complex $W_{0}(L, \mathfrak{h})_{q}$ is discussed in 4.70.

The isomorphism of the algebras $H\left(W_{s}(L, \mathfrak{h})_{q}\right)$ for all $s \geqq 0$ and all Lie algebras $L$ over $g$ is established in Section 5 . The DG-algebra $W_{s}(L, \mathfrak{h})_{q}$ defined in 5.3 is the quotient of $W_{s}(L, \mathfrak{h})$ by the ideal $F_{s}^{2(q+1)}(\mathfrak{g}) W_{s}(L, \mathfrak{h})$ of an appropriate filtration $F_{s}(\mathfrak{g})$ on $W_{s}$ (defined in 5.1). Theorem 5.5 follows from the previously established facts by a general spectral sequence argument. It also follows that the additive map $\lambda$ in 
(1.3) induces an isomorphism $\lambda_{*}: H\left(W(\mathfrak{g}, \mathfrak{h})_{q}\right) \rightarrow H\left(W_{1}(\mathfrak{g}, \mathfrak{h})_{q}\right)$, which in fact is multiplicative (Theorem 5.21).

These results are applied in Section 6 to the generalized characteristic homomorphism of a foliated bundle $P \rightarrow M$. The basic underlying fact is the filtration property 6.4 of the generalized Weil homomorphism. Note that there again the critical filtration on $W_{1}$ is $F_{1}$ and not the canonical filtration as a $\mathrm{g}$-DG-algebra. This is the geometric reason for the appearance of the filtrations $F_{s}$ on $W_{s}$ for $s \geqq 0$ in the earlier sections. The construction of $\Delta(\omega)$ in diagram (6.9) depends on a family $\omega=\left(\omega_{j}\right)$ of adapted connections $\omega_{j}$ on $P \mid U_{j}$, where $\mathscr{Q}=\left(U_{j}\right)$ is an open covering of $M$. This construction on the cohomology level is shown to be independent of the particular choice of $\omega$ (Proposition 6.11). This leads to a semi-simplicial characteristic homomorphism of the form

$$
\Delta(P)_{*}: H\left(W(\mathfrak{g}, H)_{q}\right) \rightarrow H_{\mathrm{DR}}(M),
$$

in 6.17. The target is the De Rham cohomology of $M$, viewed as hypercohomology of $M$ with coefficients in the sheaf complex $\Omega_{M}^{*}$ of forms on $M$ in the sense of Grothendieck. The main properties of this map are summarized in Theorem 6.21. We refer also to [16] for an elaboration of the functorial nature of this construction. The paper concludes with comments on specific geometric situations where this theory applies.

2. Semi-simplicial Weil algebras. For the discussion of semi-simplicial objects (ss objects) in various categories we need the basic category $\Delta$ with objects $\Delta_{l}=\{0, \cdots, l\}, l \geqq 0$; face maps

$$
\varepsilon_{i}^{l}: \Delta_{l} \rightarrow \Delta_{l+1} ; \varepsilon_{i}^{l}(k)=\left\{\begin{array}{ll}
k & 0 \leqq k<i \\
k+1 & i \leqq k \leqq l
\end{array}\right\}
$$

for $i=0, \cdots, l+1$; and degeneracy maps

$$
\mu_{j}^{l}: \Delta_{l+1} \rightarrow \Delta_{l} ; \mu_{j}^{l}(k)=\left\{\begin{array}{ll}
k & 0 \leqq k \leqq j \\
k-1 & j<k \leqq l+1
\end{array}\right\}
$$

for $j=0, \cdots, l$; which by composition generate all weakly monotone increasing maps $\Delta_{p} \rightarrow \Delta_{q}$. These face and degeneracy maps satisfy the relations

$$
\begin{array}{ll}
\varepsilon_{i}^{l} \circ \varepsilon_{j}^{l-1}=\varepsilon_{j+1}^{l} \circ \varepsilon_{i}^{l-1} & i \leqq j \\
\mu_{j}^{l-1} \circ \mu_{i}^{l}=\mu_{i}^{l-1} \circ \mu_{j+1}^{l} & i \leqq j
\end{array}
$$




$$
\mu_{j}^{l} \circ \varepsilon_{i}^{l}= \begin{cases}\varepsilon_{i}^{l-1} \circ \mu_{j-1}^{l-1} & i<j \\ 1 & i=j, j+1 . \\ \varepsilon_{i-1}^{l-1} \circ \mu_{j}^{l-1} & i>j+1\end{cases}
$$

A ss object in a category $\mathscr{C}$ is a contravariant functor $\Delta m \mathscr{C}$, a co-ss object a covariant functor $\Delta m \mathscr{C}$ (see e.g., [23] for standard terminology). We need several such constructions in this paper.

First we consider finite-dimensional Lie algebras over a field of characteristic zero. For every such Lie algebra $L$ there is an associated ss Lie algebra

$$
\Delta L=\left(L_{l}\right)_{l \geqq 0} ; L_{l}=L^{l+1}=\underbrace{L \times \cdots \times L}_{l+1 \text { factors }}
$$

with face and degeneracy operators given by

$$
\begin{aligned}
& \varepsilon_{i}^{l}: L_{l+1} \rightarrow L_{l} ; \varepsilon_{i}^{l}\left(x_{0}, \cdots, x_{l+1}\right)=\left(x_{0}, \cdots, \hat{x}_{i}, \cdots, x_{l+1}\right) \\
& \mu_{j}^{l}: L_{l+1} \rightarrow L_{l} ; \mu_{j}^{l}\left(x_{0}, \cdots, x_{l}\right)=\left(x_{0}, \cdots, x_{j}, x_{j}, \cdots, x_{l}\right)
\end{aligned}
$$

for $i=0, \cdots, l+1 ; j=0, \cdots, l$. These operators satisfy the relations (dual to the relations (2.1) (2.2) (2.3))

$$
\begin{aligned}
& \varepsilon_{i}^{l-1} \circ \varepsilon_{j+1}^{l}=\varepsilon_{j}^{l-1} \circ \varepsilon_{i}^{l} \quad i \leqq j \\
& \mu_{j+1}^{l} \circ \mu_{i}^{l-1}=\mu_{i}^{l} \circ \mu_{j}^{l-1} \quad i \leqq j \\
& \varepsilon_{i}^{l} \circ \mu_{j}^{l}=\left\{\begin{array}{cl}
\mu_{j-1}^{l-1} \circ \varepsilon_{i}^{l-1} & i<j \\
1 & i=j, j+1 \\
\mu_{j}^{l-1} \circ \varepsilon_{i-1}^{l-1} & i>j+1
\end{array}\right\} .
\end{aligned}
$$

This construction is clearly covariant functorial in $L$.

Consider now the Weil algebra construction

$$
W(L)=\wedge L^{*} \otimes S L^{*}
$$

for a Lie algebra $L$ (see [5] or [16], p. 54 for the definition). It is a commutative DG-algebra which is contravariant functorial for Lie algebra homomorphisms $L \rightarrow L^{\prime}$. Applied to $\Delta L$ it leads to a co-ss commutative DG-algebra

$$
W(\Delta L)=\left(W\left(L_{l}\right)\right)_{l \geqq 0}, \quad W\left(L_{l}\right)=W\left(L^{l+1}\right) \cong W(L)^{\otimes l+1}
$$

with face and degeneracy operators

$$
\begin{aligned}
& W\left(\varepsilon_{i}^{l}\right): W\left(L_{l}\right) \rightarrow W\left(L_{l+1}\right) \\
& W\left(\mu_{j}^{l}\right): W\left(L_{l+1}\right) \rightarrow W\left(L_{l}\right)
\end{aligned}
$$

induced by the dual maps 


$$
\begin{aligned}
& \left(\varepsilon_{i}^{l}\right)^{*}: L_{l}^{*} \rightarrow L_{l+1}^{*} ;\left(\varepsilon_{i}^{l}\right)^{*}\left(\alpha_{0}, \cdots, \alpha_{l}\right)=\left(\alpha_{0}, \cdots, \alpha_{i-1}, 0, \alpha_{i}, \cdots, \alpha_{l}\right) \\
& \left(\mu_{j}^{l}\right)^{*}: L_{l+1}^{*} \rightarrow L_{l}^{*} ;\left(\mu_{j}^{l}\right)^{*}\left(\alpha_{0}, \cdots, \alpha_{l+1}\right)=\left(\alpha_{0}, \cdots, \alpha_{j}+\alpha_{j+1}, \cdots, \alpha_{l+1}\right)
\end{aligned}
$$

for $i=0, \cdots, l+1, j=0, \cdots, l$ on the Lie algebras involved. We still denote $W\left(\varepsilon_{i}^{l}\right)=\varepsilon_{i}^{l}$ and $W\left(\mu_{j}^{l}\right)=\mu_{j}^{l}$. These operators satisfy then the relations (2.1) (2.2) (2.3). The evaluation formulas for these operators are

$$
\begin{aligned}
& \varepsilon_{i}^{l}\left(a_{0} \otimes \cdots \otimes a_{l}\right)=a_{0} \otimes \cdots \otimes a_{i-1} \otimes 1 \otimes a_{i} \otimes \cdots \otimes a_{l} \\
& \mu_{i}^{l}\left(a_{0} \otimes \cdots \otimes a_{l+1}\right)=a_{0} \otimes \cdots \otimes a_{i} a_{i+1} \otimes \cdots \otimes a_{l+1} .
\end{aligned}
$$

It will be necessary to consider instead of Lie algebras $L$ the category $\mathscr{L}_{\mathrm{g}}$ of Lie algebras $L$ over a fixed Lie algebra $\mathrm{g}$. The objects are Lie algebra inclusions $\mathrm{g} \stackrel{i_{L}}{\rightarrow} L$, and the morphisms are Lie homomorphisms $L^{\prime} \stackrel{\varphi}{\rightarrow} L$ making the diagram

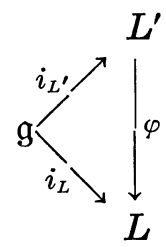

commutative. E.g.,

$$
W(L) \stackrel{W\left(i_{L}\right)}{\longrightarrow} W(\mathrm{~g})
$$

plays now the role of the Weil algebra for such an object $\mathrm{g} \stackrel{i_{L}}{\rightarrow} L$ of $\mathscr{L}_{8}$. For the ss Lie algebra $\Delta L$ over $g$ the co-ss DG-algebra $W(\Delta L)$ is then a family of Weil algebras over $W(\mathfrak{g})$.

Next we concentrate our attention on the $g$-DG-structure on the Weil algebra $W(L)$. We recall the concept of a $g$-DG-algebra $A$ (see [5] and [16], p. 46). This is a differential graded algebra $A$, which is equipped with derivations $i(x): A^{*} \rightarrow A^{-1}$ of degree -1 and derivations $\theta(x)$ : $A^{*} \rightarrow A^{\cdot}$ of degree 0 for every $x \in \mathfrak{g}$, such that the following conditions hold:

$$
\left\{\begin{array}{l}
i(x)^{2}=0 \text { for all } x \in \mathfrak{g} ; \\
\theta[x, y]=[\theta(x), \theta(y)] \text { for all } x, y \in \mathfrak{g} ; \\
{[\theta(x), i(y)]=i[x, y] \text { for all } x, y \in \mathfrak{g} ;} \\
\theta(x)=i(x) d+d i(x) \text { for } x \in \mathfrak{g} .
\end{array}\right.
$$

Here the commutator of derivations $D$ and $D^{\prime}$ of degree $r$ and $r^{\prime}$ is the derivation of degree $r+r^{\prime}$ given by 


$$
\left[D, D^{\prime}\right]=D D^{\prime}+(-1)^{r r^{\prime}+1} D^{\prime} D .
$$

The conditions (2.9) are equivalently expressed by saying that $A^{\cdot}$ is a differential $V(\mathfrak{g})$-algebra, where

$$
V .(\mathfrak{g})=U(\mathfrak{g}) \otimes \wedge .(\mathfrak{g})
$$

is defined via the enveloping algebra $U(\mathfrak{g})$ and the exterior algebra $\wedge$.(g) with a twisted tensor multiplication as in [16], p. 96. The operators $\theta(x), i(x)$ for $x \in \mathfrak{g}$ give rise to maps $V_{p} \otimes A^{*} \rightarrow A^{\cdot-p}$ characterized for $p=1$ by

$$
\begin{aligned}
& (\underline{x} \otimes 1) . a=\theta(x) a \\
& (1 \otimes x) . a=i(x) a .
\end{aligned}
$$

Here the map $\mathfrak{g} \rightarrow U(\mathfrak{g})$ is as usual denoted $x \rightarrow \underline{x}$. Homomorphisms of $\mathfrak{g}$-DG-algebras are homomorphisms of differential $V(\mathfrak{g})$-algebras.

We need also to consider differential $V(\mathfrak{g})$-modules or $\mathfrak{g}$-DG-modules. This is a DG-module $(M, d)$ with a $V(\mathfrak{g})$-action such that the Cartan formula

$$
\theta(x)=d i(x)+i(x) d
$$

holds for $x \in \mathfrak{g}$. Homomorphisms of $\mathfrak{g}$-DG-modules are homomorphisms of differential $V(\mathfrak{g})$-modules.

For a Lie algebra $L$ over $\mathfrak{g}$ the canonical $L$-DG-structure restricts to a $\mathfrak{g}$-DG-algebra structure on $W(L)$. For the ss Lie algebra $\Delta L=$ $\left(L_{l}\right)_{l \geq 0}$ over $\mathfrak{g}$ the $V(\mathfrak{g})$-action on $W\left(L_{l}\right)=W(L)^{\otimes l+1}$ is modified by the convention

$$
\begin{aligned}
(\underline{x} \otimes 1)_{.} a & =\theta(x) a \\
(1 \otimes x)_{.} a & =(-1)^{l} i(x) a
\end{aligned}
$$

for $x \in \mathfrak{g}$ and $a \in W\left(L_{l}\right)$. This turns $W(\Delta L)$ into a co-ss $\mathfrak{g}$-DG-algebra.

The construction of the ss Weil algebras is best understood in the following general context.

Let $S$ be a ss set $S=\left(S_{l}\right)_{l \geq 0}$ with face and degeneracy operators as in (2.4) to (2.6). A local system of $g$-DG-algebras $A$ on $S$ is a family

$$
\boldsymbol{A}=\left(A_{\sigma}\right), \quad \sigma \in S
$$

of $\mathrm{g}$-DG-algebras parametrized by the simplices $\sigma$ of $S$, and $\mathrm{g}$-DG-homomorphisms

$$
\begin{aligned}
& \bar{\varepsilon}_{i}^{l}(\sigma): A_{\varepsilon_{i}^{l}(\sigma)} \rightarrow A_{\sigma} \quad \text { for } \quad \varepsilon_{i}^{l}: S_{l+1} \rightarrow S_{l} \quad \text { and } \quad \sigma \in S_{l+1} \\
& \bar{\mu}_{j}^{l}(\sigma): A_{\mu_{i}^{l}(\sigma)} \rightarrow A_{\sigma} \text { for } \mu_{j}^{l}: S_{l} \rightarrow S_{l+1} \text { and } \sigma \in S_{l}
\end{aligned}
$$


with obvious composition rules (corresponding to (2.1) to (2.3)). A $\mathrm{g}$ DG-algebra $A$ determines on every ss set $S$ a constant local system also denoted $A$ with $A_{\sigma}=A$ for all $\sigma \in S$ and $\bar{\varepsilon}_{i}^{l}=\mathrm{id}, \bar{\mu}_{j}^{l}=$ id for all $i, j$.

We consider then on $S$ the $A$-valued cochains

$$
C^{*}\left(S, A^{*}\right)=\prod_{l \geq 0} C^{l}\left(S, A^{*}\right), C^{l}(S, A)=\prod_{\sigma \in S_{l}} A_{\sigma}^{*} .
$$

Together with the maps,

$$
\begin{aligned}
& \varepsilon_{i}^{l}: C^{l} \rightarrow C^{l+1}, \quad\left(\varepsilon_{i}^{l} c\right)(\sigma)=\bar{\varepsilon}_{i}^{l} c\left(\varepsilon_{i}^{l} \sigma\right) \text { for } \quad \sigma \in S_{l+1}, \quad c \in C^{l} \\
& \mu_{j}^{l}: C^{l+1} \rightarrow C^{l}, \quad\left(\mu_{j}^{l} c\right)(\sigma)=\bar{\mu}_{j}^{l} c\left(\mu_{j}^{l} \sigma\right) \text { for } \sigma \in S_{l}, \quad c \in C^{l+1}
\end{aligned}
$$

this is a co-ss $\mathrm{g}$-DG-algebra. The differential $d_{c}$ of total degree 1 is defined on $l$-cochains by the usual formula

$$
d_{C}=\delta+(-1)^{l} d_{A}
$$

where $\delta$ is the simplicial differential of bidegree $(1,0)$

$$
\delta=\sum_{i=0}^{l+1}(-1)^{i} \varepsilon_{i}^{l}: C^{l} \rightarrow C^{l+1}
$$

and $d_{A}$ is of course of bidegree $(0,1)$. The $V(\mathrm{~g})$-action is defined by

$$
\left\{\begin{array}{l}
((x \otimes 1) \cdot c)(\sigma)=\theta(x) c(\sigma) \\
((1 \otimes x) \cdot c)(\sigma)=(-1)^{l} i(x) c(\sigma)
\end{array}\right.
$$

for $c \in C^{l}, \sigma \in S_{l}$ and $x \in \mathfrak{g}$.

Proposition 2.14. $C^{*}\left(S, A^{*}\right)$ is a $\mathrm{g}$-DG-algebra.

Proof. We have defined the $g$-DG-structure. It remains to explain the Alexander-Whitney multiplication and verify the compatibility with the differential $d_{C}$. Let $C^{p, q}=C^{p}\left(S, A^{q}\right)$. Then the multiplication

$$
\mu_{C}: C^{p, q} \otimes C^{p^{\prime}, q^{\prime}} \rightarrow C^{p+p^{\prime}, q+q^{\prime}}
$$

is given by the composition

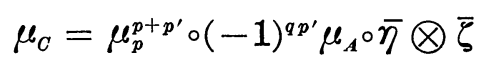

where $\mu_{A}$ is induced by the multiplication in $\boldsymbol{A}$ and

$$
\begin{aligned}
& \bar{\eta}=\varepsilon_{p+p^{\prime}+1}^{p+p^{\prime}} \cdots \circ \varepsilon_{p+1}^{p}: C^{p, q} \rightarrow C^{p+p^{\prime}+1, q} \\
& \bar{\zeta}=\varepsilon_{p}^{p+p^{\prime}} \circ \cdots \circ \varepsilon_{0}^{p^{\prime}}: C^{p^{\prime}, q^{\prime}} \rightarrow C^{p+p^{\prime}+1, q^{\prime}}
\end{aligned}
$$

are defined by the face operators in $C$. This is an associative multiplication and has all desired properties.

The g-DG-algebra $C(S, A)$ is obviously functorial for maps $f: S \rightarrow S^{\prime}$ of ss sets in the following sense. If $\boldsymbol{A}^{\prime}$ is a local system of $\mathfrak{g}$-DG-alge- 
bras on $S^{\prime}$ with pull-back $f^{*} A^{\prime}$ on $S$ given by $\left(f^{*} A^{\prime}\right)_{\sigma}=A_{f(\sigma)}^{\prime}, \sigma \in S_{l}$, $l \geqq 0$, then there is a canonical map

$$
C\left(f, \boldsymbol{A}^{\prime}\right): C\left(S^{\prime}, \boldsymbol{A}^{\prime}\right) \rightarrow C\left(S, f^{*} \boldsymbol{A}^{\prime}\right),
$$

the pull-back of cochains. If $\boldsymbol{A}$ is moreover a local system of $\mathrm{g}$-DGalgebras on $S$, and $f: f^{*} A^{\prime} \rightarrow A$ a morphism of local systems (on $S$ ) with induced cochain map

$$
C(S, \boldsymbol{f}): C\left(S, f^{*} \boldsymbol{A}^{\prime}\right) \rightarrow C(S, \boldsymbol{A}),
$$

then the composition $C(f, \boldsymbol{f})=C(S, \boldsymbol{f}) \circ C\left(f, \boldsymbol{A}^{\prime}\right)$ defines a homomorphism

$$
C(f, \boldsymbol{f}): C\left(S^{\prime}, \boldsymbol{A}^{\prime}\right) \rightarrow C(S, \boldsymbol{A})
$$

of $\mathfrak{g}$-DG-algebras.

The total cohomology of $C(S, A)$ with respect to the total differential (2.11) is denoted by $H(S ; \boldsymbol{A})$, the one with respect to the simplicial differential (2.12) is denoted $H_{\delta}(S ; \boldsymbol{A})$. The first filtration on $C(S, A)$ gives rise to a spectral sequence converging to $H(S ; \boldsymbol{A})$, whose $E_{2}^{p, q}$-term is given by $H_{\delta}^{p}\left(S, \boldsymbol{H}^{q}\left(\boldsymbol{A}^{*}\right)\right)$. Here $\boldsymbol{H}^{q}\left(\boldsymbol{A}^{*}\right)$ denotes the local system $\sigma \rightarrow$ $H^{q}\left(A_{\sigma}^{*}\right)$ on $S$.

We can apply the construction of $C(S, A)$ in particular for the ss point $S=\mathrm{Pt}$. This is the terminal object in the category of ss sets with exactly one simplex $\sigma_{l}$ in each dimension $l \geqq 0$ and canonical face and degeneracy maps. A local system of $g$-DG-algebras on $\mathrm{Pt}$ is the same as a co-ss $g$-DG-algebra. Consider for a Lie algebra $L$ over $g$ the co-ss g-DG-algebra $W(\Delta L)$, which we denote $W(\Delta L)$ when viewed as a local system on Pt.

Definition 2.18. The $\mathrm{g}$-DG-algebra

$$
W_{1}(L)=C(\mathrm{Pt}, W(\Delta L))
$$

is called the (first) ss Weil algebra of $L$.

The subscript indicates that this construction will be iterated to yield g-DG-algebras $W_{s}(L)$ for all $s \geqq 0$, where $W_{0}(L)=W(L)$. This object has of course co-ss character, but the term co-ss Weil algebra seemed overly pedantic.

The local system $W(\Delta L)$ on $\mathrm{Pt}$ assigns to $\sigma_{l}$ the algebra $W\left(L_{l}\right)=$ $W\left(L^{l+1}\right)=W(L)^{\otimes l+1} . \quad W_{1}(L)$ has the bigrading

$$
W_{1}(L)^{l, m}=\left(W(L)^{\otimes l+1}\right)^{m} .
$$

By (2.11) the differential $d_{W_{1}}$ of total degree 1 is defined on elements of $W_{1}^{l, \cdot}=W_{1}^{l}$ by 


$$
d_{W_{1}}=\delta+(-1)^{l} d_{W_{1}^{l}}
$$

where

$$
\delta=\sum_{i=0}^{l+1}(-1)^{i} \varepsilon_{i}: W_{1}^{l} \rightarrow W_{1}^{l+1} .
$$

Note that the Weil differential $d_{W_{1}^{l}}: W_{1}^{l, \cdot} \rightarrow W_{1}^{l, \cdot}$ is of bidegree $(0,1)$ and $\delta$ of bidegree $(1,0)$.

The $V(\mathrm{~g})$-action on $W_{1}^{l}$ is according to (2.13) given by

$$
\left\{\begin{array}{l}
(\underline{x} \otimes 1) . a=\theta(x) \cdot a \\
(1 \otimes x) \cdot a=(-1)^{l} i(x) a
\end{array}\right.
$$

for $a \in W_{1}^{l}$ and $x \in \mathrm{g}$. The Alexander-Whitney multiplication in $C(\mathrm{Pt}$, $W(\Delta L)$ ) turns $W_{1}(L)$ into a $\mathrm{g}$-DG-algebra. We finally note that the construction of $W_{1}(L)$ from $W(L)$ is reminiscent of the construction of the Amitsur complex of an algebra.

The construction of $W_{1}$ from $W=W_{0}$ can be iterated. The only thing to observe is that $W_{1}(L)$ is again contravariant functorial in $L$ (as Lie algebra over $\mathrm{g}$ ).

DEFINITION 2.22. The (iterated) ss Weil algebra $W_{s}(L)$ of $L$ is the g-DG-algebra

$$
W_{s}(L)=C\left(\mathrm{Pt}, W_{s-1}(\Delta L)\right), \quad s>0 .
$$

This can be expressed more formally as follows. Let $\mathscr{L}_{\mathrm{a}}$ denote as before the category of Lie algebras $L$ over the fixed Lie algebra $g$. The construction of the semi-simplicial Lie algebra associated to $L$ defines a functor $\Phi: \mathscr{L}_{8} \leadsto \Delta \mathscr{L}_{8}$ into the category $\Delta \mathscr{L}_{8}$ of ss objects of $\mathscr{L}_{8}$. Let $\mathscr{A}_{8}$ denote the category of $\mathfrak{g}$-DG-algebras. Then a contravariant functor $F: \mathscr{L}_{B} \rightarrow \mathscr{A}_{B}$ induces a contravariant functor $\Delta F: \Delta \mathscr{L}_{8} m{ }^{4} \mathscr{A}_{\mathrm{B}}$ into the category ${ }^{\triangle} \mathscr{A}_{8}$ of co-ss objects of $\mathscr{A}_{8}$. The cochain complex construction induces a functor $\Psi:{ }^{\Delta} \mathscr{A}_{B} \rightarrow \mathscr{A}_{8}$. The composition

$$
F_{1}=\Psi \circ \Delta F \circ \Phi: \mathscr{L}_{\mathrm{g}} \leadsto \mathscr{A}_{\mathrm{g}}
$$

is a contravariant functor associated to $F=F_{0}$. We can iterate this construction and define a sequence of functors $F_{s}, s \geqq 0$ by the recursion formula $F_{s+1}=\left(F_{s}\right)_{1}$. For $F=W=W_{0}$ we get the functors $W_{s}, s \geqq 0$.

The canonical projections

$$
\rho_{s}: W_{s}(L) \rightarrow W_{s}^{0}(L)=W_{s-1}(L), \quad s>0
$$

are obviously $\mathrm{g}$-DG-homomorphisms. The Weil algebra $W(L)$ is well known to be cohomologically trivial. It is not difficult to verify the 
same property for the ss Weil algebras $W_{s}(L), s>0$. But the essential feature of these algebras is that they behave cohomologically like the Weil algebra $W(L)$ in a much stronger sense. The precise statement is given in Theorem 5.5 and Corollary 5.7 of this paper. The reason for the introduction of these algebras has been explained in the introduction and is discussed again in Section 6.

3. Homotopies. The main topic of this section is a homotopy construction, which makes its appearance practically in every aspect of the theory of secondary characteristic classes. It embodies among other things the algebraic features of the classical Weil lemma on characteristic forms in a principal bundle.

We begin by constructing for every commutative g-DG-algebra $E$. a simplicial $\mathrm{g}$-DG-algebra $\boldsymbol{E}=\left(E^{(l)}\right)_{l \geq 0}$ as follows. Let

$$
\begin{aligned}
\left(E^{(l)}\right)^{\cdot, \cdot}=\left(E^{*}\left[t_{0}, \cdots, t_{l}\right] /\left(\sum_{j=0}^{l} t_{j}-1\right)\right) & \\
\otimes & \left(\wedge^{\cdot}\left(d t_{0}, \cdots, d t_{l}\right) /\left(\sum_{j=0}^{l} d t_{j}\right)\right)
\end{aligned}
$$

where $t_{0}, \cdots, t_{l}$ are elements of degree 0 and $d t_{0}, \cdots, d t_{l}$ elements of degree 1. The algebra $E^{(l)}$ is considered attached to the standard $l$ simplex $\Delta^{(l)}=\left\{\left(t_{0}, \cdots, t_{l}\right) \mid \sum_{j=0}^{l} t_{j}=1\right.$ and $\left.t_{j} \geqq 0\right\}$. The differential $d_{E}$ is defined by sending $d t_{j}$ to 0 and by the formula

$$
\begin{gathered}
d_{E}\left(e\left(t_{0}, \cdots, t_{l}\right) \otimes \omega^{k}\right)=\left(d_{E} e\right)\left(t_{0}, \cdots, t_{l}\right) \otimes \omega^{k} \\
+(-1)^{\operatorname{deg} e} \sum_{j=0}^{l} \frac{\partial}{\partial t_{j}} e\left(t_{0}, \cdots, t_{l}\right) \otimes d t_{j} \wedge \omega^{k} .
\end{gathered}
$$

The face maps $\varepsilon_{j}^{l}: \Delta^{(l)} \rightarrow \Delta^{(l+1)}$ given by

$$
\varepsilon_{j}^{l}\left(t_{0}, \cdots, t_{l}\right)=\left(t_{0}, \cdots, t_{j-1}, 0, t_{j}, \cdots, t_{l}\right)
$$

induce maps $\varepsilon_{j}^{l}: E^{(l+1)} \rightarrow E^{(l)}$ by sending

$$
\begin{aligned}
& t_{i}, d t_{i} \rightarrow t_{i}, d t_{i} \text { for } 0 \leqq i<j \\
& t_{j}, d t_{j} \rightarrow 0, \\
& t_{i}, d t_{i} \rightarrow t_{i-1}, d t_{i-1} \text { for } j<i \leqq l+1 .
\end{aligned}
$$

This turns $\boldsymbol{E}$ into a simplicial DG-algebra. Modifying the $i$-action on $E^{-l}$ by the factor $(-1)^{l}$ and extending $i, \theta$ to derivations of degree $-1,0$ of $E^{l}$ by $i\left(t_{j}\right)=0, i\left(d t_{j}\right)=0, \theta\left(t_{j}\right)=0, \theta\left(d t_{j}\right)=0$, this turns $E$ into a simplicial $\mathrm{g}$-DG-algebra.

A boundary operator $\partial: E^{(l+1)} \rightarrow E^{(l)}$ is defined by 


$$
\partial=\sum_{j=1}^{l+1}(-1)^{j} \varepsilon_{j}^{l}
$$

A $\operatorname{map} \pi_{*}^{(l)}: E^{(l) \cdot} \rightarrow E^{\cdot-l}$ is defined by integration over the standard simplex $\Delta^{(l)}$ as follows. If

$$
v=e^{m}\left(t_{0}, \cdots, t_{l}\right) \otimes \nu_{0}^{l} \in\left(E^{m}\left[t_{0}, \cdots, t_{l}\right] /\left(\sum_{j=0}^{l} t_{j}-1\right)\right) \otimes d t_{1} \wedge \cdots \wedge d t_{l}
$$

with $\nu_{j}^{l}=d t_{0} \wedge \cdots \wedge d \hat{t}_{j} \wedge \cdots \wedge d t_{l}, j=0, \cdots, l$, then we define

$$
\pi_{*}^{(l)} v=(-1)^{m l} \int_{\Delta(l)} v=(-1)^{m l} \int_{\Delta^{(l)}} e\left(t_{0}, \cdots, t_{l}\right) \otimes \nu_{0}^{l} \in E^{m} ;
$$

and on $\left(E\left[t_{0}, \cdots, t_{l}\right] /\left(\sum_{j=0}^{l} t_{j}-1\right)\right) \otimes\left(\wedge^{q}\left(d t_{0}, \cdots, d t_{l}\right) /\left(\sum_{j=0}^{l} d t_{j}\right)\right)$ for $q<l$, the $\operatorname{map} \pi_{*}^{(l)}$ is defined to be zero. Then $\pi_{*}^{(l)}$ is a $V(\mathfrak{g})$-module map.

With the preceding definitions we have the following result.

LEMMA 3.5. (Simplicial Stokes formula). On $E^{(l+1)}$

$$
\pi_{*}^{(l+1)} d_{E}+(-1)^{l} d_{E^{\circ}} \pi_{*}^{(l+1)}=\pi_{*}^{(l)} \circ \partial, l \geqq 0 .
$$

Proof. For $v=e^{m}\left(t_{0}, \cdots, t_{l+1}\right) \otimes \omega^{k} \in\left(E^{(l+1)}\right)^{m, k}$ both sides of the equation in 3.5 map $v$ into zero except for $k=l, l+1$. For $k=l+1$, it is sufficient to consider elements of the form $v=e^{m}\left(t_{0}, \cdots, t_{l+1}\right) \otimes \nu_{0}^{l+1}$. Clearly $\partial\left(1 \otimes \nu_{0}\right)=0$ and hence $\partial(v)=0$. On the other hand we have by (3.2)

$$
\pi_{*}^{(l+1)}\left(d_{E^{(l+1)}} v\right)=\pi_{*}^{(l+1)}\left\{d_{E} e^{m} \otimes \nu_{0}^{l+1}+(-1)^{m} \sum_{j=0}^{l} \frac{\partial}{\partial t_{j}} e^{m} \otimes d t_{j} \wedge \nu_{0}^{l+1}\right\}
$$

From (3.4) it follows that

(3.6) $\quad \pi_{*}^{(l+1)}\left(d_{E} e^{m}\left(t_{0}, \cdots, t_{l+1}\right) \otimes \omega^{k}\right)=(-1)^{l+1} d_{E} \pi_{*}^{(l+1)}\left(e^{m}\left(t_{0}, \cdots, t_{l+1}\right) \otimes \omega^{k}\right)$.

Using $d t_{j} \wedge \nu_{0}^{l+1}=0$ for $j=1, \cdots, l+1$ we have therefore

$$
\pi_{*}^{(l+1)}\left(d_{E^{(l+1)}} v\right)+(-1)^{l} d_{E} \pi_{*}^{(l+1)}(v)=0 \text {. }
$$

For $k=l$, it is sufficient to check (3.5) for elements of the form $v=e^{m} \otimes$ $\omega_{\alpha}^{l}$, where $\omega_{\alpha}^{l}=d t_{1} \wedge \cdots \wedge \widehat{d t}_{\alpha} \wedge \cdots \wedge d t_{l+1}, \alpha=1, \cdots, l+1$. Then

$$
\begin{gathered}
\pi_{*}^{(l+1)}\left(d_{E^{(l+1)}} v\right)=\pi_{*}^{(l+1)}\left(d_{E} e \otimes \omega_{\alpha}^{l}\right)+(-1)^{m} \sum_{j=0}^{l+1} \pi_{*}^{(l+1)}\left(\frac{\partial}{\partial t_{j}} e\left(t_{0}, \cdots, t_{l+1}\right) \otimes d t_{j} \wedge \omega_{\alpha}^{l}\right) \\
=(-1)^{l+1} d_{E^{*}} \pi_{*}^{(l+1)}(v)+(-1)^{m}(-1)^{(l+1) m} \sum_{j} \int_{A^{(l+1)}} \frac{\partial}{\partial t_{j}} e \otimes d t_{j} \wedge \omega_{\alpha}^{l},
\end{gathered}
$$

where we have used (3.6). By the classical Stokes formula, we have then 


$$
\begin{aligned}
& \pi_{*}^{(l+1)}\left(d_{E^{(l+1)}} v\right)=(-1)^{l+1} d_{E} \pi_{*}^{(l+1)}(v)+(-1)^{l m} \int_{\partial \Delta}(l+1) \\
& =(-1)^{l+1} d_{E} \pi_{*}^{(l+1)}(v)+(-1)^{l m} \sum_{j=0}^{l+1}(-1)^{j} \int_{\varepsilon_{j}^{l} d^{(l)}} e \otimes \omega_{\alpha}^{l} \\
& =(-1)^{l+1} d_{E} \pi_{*}^{l(+1)}(v)+(-1)^{l m} \sum_{j=0}^{l+1}(-1)^{j} \int_{\Delta^{(l)}} \varepsilon_{j}^{l}\left(e \otimes \omega_{\alpha}^{l}\right) \\
& =(-1)^{l+1} d_{E} \pi_{*}^{(l+1)}(v)+\pi_{*}^{(l)}(\partial v) .
\end{aligned}
$$

Actually the term $d_{E} \pi_{*}^{(l+1)}(v)=0$, since $v \in E^{(l+1) m, l}$. This finishes the proof of 3.5.

Next we consider commutative g-DG-algebras with connections. Recall that a connection in $E^{*}$ (also $g$-connection in $E^{*}$ ) is given by a multiplicative homomorphism $\wedge \mathrm{g}^{*} \stackrel{\omega}{\rightarrow} E$ of degree 0 which is a homomorphism of $V(\mathrm{~g})$-algebras (see [5] and [16], p. 97). Such a map is characterized by its restriction $\mathrm{g}^{*} \rightarrow E^{1}$ and extends to a unique homomorphism of differential $V(\mathfrak{g})$-algebras $k(\omega): W^{\cdot}(\mathfrak{g}) \rightarrow E^{\cdot}$ (the Weil homomorphism of $\omega)$ making the diagram

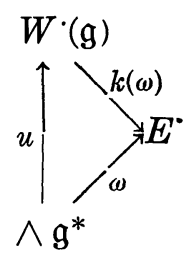

commutative (see [16], p. 57 and also the refinement in Proposition 4.7 below). The canonical map $u: \wedge \mathfrak{g}^{*} \rightarrow W(\mathfrak{g})$ is a universal connection (extending to the identity on $W(\mathfrak{g})$ ). We shall use the term connection indiscriminately for the connection map $\omega$ and its Weil homomorphism $k(\omega)$. Thus e.g., id: $W(\mathrm{~g}) \rightarrow W(\mathrm{~g})$ is the universal connection in $W(\mathrm{~g})$.

Let $E$ - again be a commutative g-DG-algebra. For any set of $l+1$ connections $\omega_{j}: \wedge \mathfrak{g}^{*} \rightarrow E$. with Weil homomorphisms

$$
k\left(\omega_{j}\right): W^{*}(\mathfrak{g}) \rightarrow E ; j=0, \cdots, l,
$$

let $\sigma=(0, \cdots, l)$ and consider

$$
k\left(\omega^{o}\right): W(\mathfrak{g}) \rightarrow E^{(l)},
$$

the Weil homomorphism of the connection in $E^{(l)}$ determined by

$$
\begin{aligned}
& \omega^{\sigma}: \mathrm{g}^{*} \rightarrow\left(E^{(l)}\right)^{1} \cdot \\
& \alpha \mapsto \sum_{j=0}^{l} t_{j} \omega_{j}(\alpha), \quad \text { where } \sum_{j=0}^{l} t_{j}=1 .
\end{aligned}
$$


The composition of $k\left(\omega^{\sigma}\right)$ with $\pi_{*}^{(l)}$ defined in (3.4)

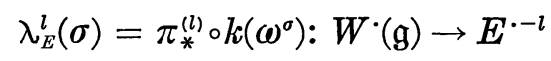

is a $V(\mathfrak{g})$-module map of degree $-l$. We also use the notation $\lambda_{E}^{l}(\sigma)=$ $\lambda_{E}^{l}\left(\omega_{0}, \cdots, \omega_{l}\right)$.

Let $\varepsilon_{j}^{l}(\sigma)=(0, \cdots, \hat{j}, \cdots, l+1)$. The boundary $\partial \sigma$ is given by

$$
\partial \sigma=\sum_{j=0}^{l+1}(-1)^{j} \varepsilon_{j}^{l}(\sigma) .
$$

Proposition 3.11. The maps $\lambda_{E}^{l}(\sigma)$ defined for all sets of $l+1$ connections, $l \geqq 0$ satisfy the following properties:

$$
\begin{cases}(\text { i }) & \lambda_{E}^{l+1}(\sigma) \circ d_{W}+(-1)^{l} d_{E^{\circ}} \lambda_{E}^{l+1}(\sigma)=\lambda_{E}^{l}(\partial \sigma) ; \\ \text { (ii }) & \lambda_{E}^{l}(\sigma) w=0 \text { for } w \in W^{q, 2 p}, l>p ; \\ \text { (iii) } & \lambda_{E}^{0}(j)=k\left(\omega_{j}\right) ; \\ \text { (iv) } & \lambda_{E}^{1}(0,1) \tilde{\alpha}=\left(\omega_{1}-\omega_{0}\right) \alpha \text { for } \alpha \in \mathfrak{g}^{*}, \sigma=(0,1), \omega=\left(\omega_{0}, \omega_{1}\right) .\end{cases}
$$

Proof. The proof of (i) follows from the Stokes formula in Lemma 3.5. Property (iii) is immediate from the definition. To verify (ii) we need to evaluate for an $l$-simplex $\sigma$ the curvature in $E^{(l)}$

$$
k\left(\omega^{\sigma}\right) \tilde{\alpha}=d_{E}(l) \omega^{\sigma} \alpha-\omega^{\sigma} d_{\wedge} \alpha
$$

for $\alpha \in \mathfrak{g}^{*}$. If we use $\sum_{j=0}^{l} t_{j}=1$, then

$$
\omega^{\sigma}=\omega_{0}+\sum_{j=1}^{l} t_{j}\left(\omega_{j}-\omega_{0}\right) .
$$

Using (3.2) and the identity (3.6) in [16], (3.13) takes the form

$$
k\left(\omega^{\sigma}\right) \tilde{\alpha}=d_{E} \omega^{o}(\alpha)+\frac{1}{2} \alpha\left[\omega^{o}, \omega^{o}\right]-\sum_{j=1}^{l} \alpha\left(\omega_{j}-\omega_{0}\right) \otimes d t_{j} .
$$

It follows by multiplicativity of $k\left(\omega^{o}\right)$ that for $w \in W^{q, 2 p}$ with $p<l$ property (iii) holds. Note that for $l=1, \sigma=(0,1), \omega=\left(\omega_{0}, \omega_{1}\right)$ we get from (3.14) in particular with $t_{0}=1-t, t_{1}=t$

$$
\begin{gathered}
k\left(\omega^{(0,1)}\right) \tilde{\alpha}=(1-t) k\left(\omega_{0}\right) \tilde{\alpha}+t k\left(\omega_{1}\right) \tilde{\alpha}-\frac{1}{2} t \alpha\left[\omega_{1}-\omega_{0}, \omega_{1}-\omega_{0}\right] \\
+\frac{1}{2} t^{2} \alpha\left[\omega_{1}-\omega_{0}, \omega_{1}-\omega_{0}\right]-\alpha\left(\omega_{1}-\omega_{0}\right) \otimes d t .
\end{gathered}
$$

Property (iv) finally follows from (i), evaluated at $\alpha \in W^{1,0}=\wedge^{1} \mathfrak{g}^{*}$ and property (ii).

REMARK 3.16. For $\sigma=(0,1)$ and $\omega=\left(\omega_{0}, \omega_{1}\right)$ formula (i) reads

$$
\lambda_{E}^{1}(0,1) d_{W}+d_{E} \lambda_{E}^{1}(0,1)=\lambda_{E}^{0}(\partial(0,1))=k\left(\omega_{1}\right)-k\left(\omega_{0}\right)
$$


where the last equality follows from (iii). But for $\Phi \in I(G)$ we have $d_{W} \Phi=0$ (see [16], p. 60), so that

$$
d_{E} \lambda_{E}^{1}(0,1) \Phi=\left(k\left(\omega_{1}\right)-k\left(\omega_{0}\right)\right) \Phi \text { for } \Phi \in I(G) \text {. }
$$

This is the Weil lemma for the characteristic homomorphisms associated to two connections $\omega_{r}, \omega_{1}$ in $E$ (classically the De Rham complex $\Omega^{\cdot}(P)$ of global forms on a principal bundle $P$ with structural group $G$ ). More generally the map $\lambda_{E}^{1}(0,1)$ is a homotopy between $k\left(\omega_{0}\right)$ and $k\left(\omega_{1}\right)$.

Next we generalize the construction of the maps $\lambda_{E}^{l}$ to sets of $l+1$ connections in a local system of $\mathfrak{g}$-DG-algebras. For this purpose we restrict ourselves to ss sets with the following property. Let $\Lambda$ be a set and consider the ss set $\left(\Lambda^{l+1}\right)_{l \geqq 0}$ of simplices in $\Lambda$. The face and degeneracy maps are given by

$$
\begin{aligned}
\Lambda^{l+2}=\operatorname{Map}\left(\Delta_{l+1} ; \Lambda\right) & \rightarrow \Lambda^{l+1}=\operatorname{Map}\left(\Delta_{l}, \Lambda\right) \\
\sigma & \mapsto \sigma \circ \varepsilon_{i}^{l} \\
\Lambda^{l+1}=\operatorname{Map}\left(\Delta_{l}, \Lambda\right) & \rightarrow \Lambda^{l+2}=\operatorname{Map}\left(\Delta_{l+1}, \Lambda\right) \\
\sigma & \mapsto \sigma \circ \mu_{j}^{l}
\end{aligned}
$$

and are again denoted by $\varepsilon_{i}^{l}, \mu_{j}^{l}, i=0, \cdots, l+1, j=0, \cdots, l$. We assume in the following that $S=\left(S_{l}\right)_{l \geqq 0}$ is a ss subset of $\left\{\Lambda^{l+1}\right\}_{l \geqq 0}$, i.e., $S_{l} \subset \Lambda^{l+1}$ and the sets $S_{l}$ are closed under the maps $\varepsilon_{i}$ and $\mu_{j}$. Let $E^{\cdot}$ be a local system of commutative $\mathfrak{g}$-DG-algebras on $S$ (see Section 2). Let $\omega=\left(\omega_{j}\right)_{j \in S_{0}}$ be a family of $\mathfrak{g}$-connections in $E$, i.e., $\omega_{j}: \wedge \mathfrak{g}^{*} \rightarrow E_{j}$ for $j \in S_{0}$. For any $l$-simplex $\sigma=\left(i_{0}, \cdots, i_{l}\right) \in S_{l}$ there are then connections $k\left(\omega_{i_{j}}\right): W(\mathrm{~g}) \rightarrow E_{i_{j}} \stackrel{\text { can }}{\longrightarrow} E_{\sigma}$. We have by (3.8) and (3.4) maps

$$
\begin{gathered}
\pi_{*}^{(l)}(\sigma): E_{\sigma}^{(l)} \rightarrow E_{\sigma}^{\cdot-l} \\
k\left(\omega^{\sigma}\right): W(\mathfrak{g})^{\bullet} \rightarrow E_{\sigma}^{(l)} .
\end{gathered}
$$

For $\sigma \in S_{l+1}$ we have thus with self-explanatory notations a commutative diagram

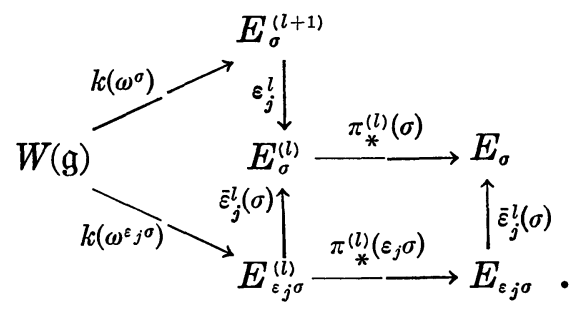

We define for every $\sigma \in S_{l}$

$$
\lambda_{E}^{l}(\sigma) \equiv \pi_{*}^{(l)}(\sigma) \circ k\left(\omega^{\sigma}\right): W \cdot(\mathfrak{g}) \rightarrow E_{\sigma}^{\cdot-l} .
$$


Applying the simplicial Stokes formula 3.5, which holds for every $\sigma$, and diagram 3.20 , we obtain

$$
\begin{aligned}
& \lambda_{E}^{l+1}(\sigma) \circ d_{W}+(-1)^{l} d_{E_{\sigma}} \circ \lambda_{E}^{l+1}(\sigma) \\
& \quad=\sum_{j=0}^{l+1}(-1)^{j} \tilde{\varepsilon}_{j}^{l}(\sigma) \lambda_{E}^{l}\left(\varepsilon_{j}^{l} \sigma\right)=\left(\delta \lambda_{E}^{l}\right)(\sigma), \text { for } \quad \sigma \in S_{l+1} .
\end{aligned}
$$

The family $\lambda_{E}^{l}(\sigma), \sigma \in S_{l}$ defines a linear map of degree zero

$$
\lambda_{E}^{l}: W(\mathfrak{g}) \rightarrow C^{l}(S, E), \quad l \geqq 0
$$

by the formula

$$
\lambda_{E}^{l}(w) \sigma=\lambda_{E}^{l}(\sigma) w, \quad \sigma \in S_{l}, \quad w \in W(\mathfrak{g}) .
$$

The family $\lambda_{E}^{l}, l \geqq 0$ defines by Proposition 3.11, part (ii) a total map

$$
\lambda_{E}^{\cdot}=\left(\lambda_{E}^{l}\right)_{l \geqq 0}: W(\mathrm{~g})^{\cdot} \rightarrow C^{\cdot}\left(S, E^{*}\right)
$$

which by (3.22) commutes with $d_{W}$ and the total differential $d_{C}$ in $C^{*}(S$, $\left.\boldsymbol{E}^{*}\right)$. Thus $\lambda_{E}^{*}$ is a map of differential $V(\mathrm{~g})$-modules (see (2.13) for the $i$-action). The other properties of $\lambda_{E}^{l}$ translate similarly and we have the following result.

THEOREM 3.25. Let $\boldsymbol{E}$ be a local system of g-DG-algebras on the ss set $S$, and $\omega=\left(\omega_{j}\right)_{j \in S_{0}}$ a family of connections in $\boldsymbol{E}$ as above. Then there exists a canonical map $\lambda_{E}: W(\mathfrak{g})^{*} \rightarrow C^{*}\left(S, E^{*}\right)$ of degree zero satisfying the following properties:

(i) $\lambda_{E}^{*}$ is a map of differential $V(\mathfrak{g})$-modules;

(ii) $\lambda_{E}^{l}(w)=0$, for $w \in W^{q, 2 p}(\mathrm{~g}), l>p$;

(iii) $\lambda_{E}^{0}: W(\mathfrak{g}) \rightarrow C^{0}(S, E)$ is given by $\lambda_{E}^{0}(w)_{j}=k\left(w_{j}\right)$ for $j \in S_{0}$, $w \in W(\mathrm{~g})$;

(iv) $\lambda_{E}^{1}(\widetilde{\alpha})=\delta \lambda_{E}^{0}(\alpha)$ for $\alpha \in \wedge^{1} \mathfrak{g}^{*}$.

This construction can in particular be applied to the ss Weil algebra

$$
W_{1}(L)^{\bullet}=C^{*}(\mathrm{Pt}, W(\Delta L))
$$

as defined in (2.18) for any Lie algebra $L$ over $\mathfrak{g}$. The connection defined in $W(\Delta L)_{0}=W(L)$ for the unique 0 -simplex of $\mathrm{Pt}$ is the universal connection $u: \wedge L^{*} \rightarrow W(L)$. In $W\left(L^{l+1}\right)=W(\Delta L)_{l}$ we have the $l+1$ connections $u_{j}: \wedge L^{*} \rightarrow W\left(L^{l+1}\right) \cong W(L)^{\otimes l+1}$ given by inclusion into the $j^{\text {th }}$ factor. Theorem 3.25 translates then to the following statement.

THEOREM 3.26. Let $L$ be a Lie algebra over g. There exists a canonical $V(\mathfrak{g})$-module map $\lambda: W(L) \rightarrow W_{1}(L)$ of degree zero defined by linear maps $\lambda^{l}: W(L)^{\cdot} \rightarrow W\left(L^{l+1}\right)^{\cdot-l}$ of degree $-l(l \geqq 0)$ and satisfying the following properties: 
(i ) $\lambda^{l+1} \circ d_{W}+(-1)^{l} d_{W_{1}^{l+1} \circ} \lambda^{l+1}=\sum_{j=0}^{l+1}(-1)^{j} \varepsilon_{j}^{l} \lambda^{l}, l \geqq 0$; i.e., $\lambda$ is a map of differential $V(\mathfrak{g})$-modules;

(ii) $\rho_{1} \circ \lambda=\mathrm{id}_{W}$;

(iii) $\lambda^{l}(w)=0$ for $w \in W(L)^{q, 2 p}, l>p$.

(iv) $\lambda^{1} \tilde{\alpha}=\delta \alpha$ for $\alpha \in \wedge^{1} \mathrm{~g}^{*}$.

We observe that $\lambda$ is universal in the sense that it determines all the mappings $\lambda_{E}$ via a certain coefficient map. Let $f: S \rightarrow$ Pt denote the unique semi-simplicial morphism and denote $\boldsymbol{W}(\Delta \mathfrak{g})_{S}=f^{*} \boldsymbol{W}(\Delta \mathfrak{g})$, the pull-back of $W(\Delta \mathfrak{g})$ to $S$ (see Section 2). Given a family $\omega=\left(\omega_{j}\right)$ of $\mathfrak{g}$ connections on $\boldsymbol{E} \cdot$ as before, we construct a coefficient map

$$
k(\omega): W(\Delta \mathfrak{g})_{S} \rightarrow \boldsymbol{E}^{\cdot}
$$

as follows. For every $\sigma=\left(i_{0}, \cdots, i_{l}\right) \in S_{l}, l \geqq 0$ the $l+1$ connections $\omega_{i j}: \wedge \mathfrak{g}^{*} \rightarrow E_{i_{j}} \stackrel{\text { can }}{\longrightarrow} E_{\sigma}$ determine a unique $V(\mathfrak{g})$-module map

$$
\omega_{\sigma}=\left(\omega_{i_{0}}, \cdots, \omega_{i_{l}}\right): \wedge\left(\mathrm{g}^{l+1}\right)^{*} \rightarrow E_{\sigma}
$$

which extends by (4.7) below to a unique $\mathrm{g}$-DG algebra homomorphism

$$
k\left(\omega_{\sigma}\right): W\left(\mathfrak{g}^{l+1}\right) \rightarrow E_{\sigma} .
$$

Using the canonical isomorphism $W\left(\mathfrak{g}^{l+1}\right) \cong W(\mathfrak{g})^{\otimes l+1}$ we may identify $k\left(\omega_{o}\right)$ with the algebra sum of the Weil homomorphisms $k\left(\omega_{i_{j}}\right)$ :

$$
k\left(\omega_{\sigma}\right)=\left(k\left(\omega_{i_{0}}\right), \cdots, k\left(\omega_{i_{l}}\right)\right) .
$$

As $\left(W(\Delta \mathfrak{g})_{s}\right)_{o}=W\left(\mathfrak{g}^{l+1}\right)$ for $\sigma \in S_{l}$, and as the maps $k\left(\omega^{o}\right)$ are evidently compatible with face and degeneracy maps, we obtain a coefficient map

$$
k(\boldsymbol{\omega})=\left(k\left(\omega_{\sigma}\right)\right)_{\sigma \in S}: W(\Delta \mathfrak{g})_{S} \rightarrow C^{*}\left(S, E^{*}\right) .
$$

By the general construction in (2.17) this determines a $g$-DG-algebra homomorphism

$$
k_{1}(\boldsymbol{\omega}) \equiv C(f, k(\omega)): \quad W_{1}(\mathfrak{g})=C^{\bullet}(\mathrm{Pt}, W(\Delta \mathfrak{g})) \rightarrow C^{\bullet}\left(S, E^{\cdot}\right) .
$$

As the construction of $\lambda_{E}$ is compatible with coefficient maps, we obtain the formula

$$
\lambda_{E}(\omega)=k_{1}(\omega) \circ \lambda,
$$

i.e., the diagram

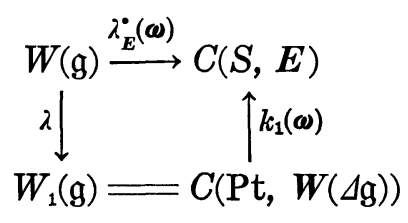


is commutative. The construction of $k_{1}(\omega)$ can be extended to $W_{s}(\mathfrak{g}), s \geqq$ 1 as follows. Let $T$ be another simplicial set and let $\omega_{k}=\left(\omega_{k, j}\right)_{j \in S_{0}}$ be a family of connections for $E$ for every $k \in T_{0}$. Then for $\omega=\left(\omega_{k}\right)_{k \in T_{0}}$ we define $k_{2}(\boldsymbol{\omega}): W_{2}(\mathrm{~g}) \rightarrow C \cdot(T, C \cdot(S, \boldsymbol{E}))$ by the following commutative diagram

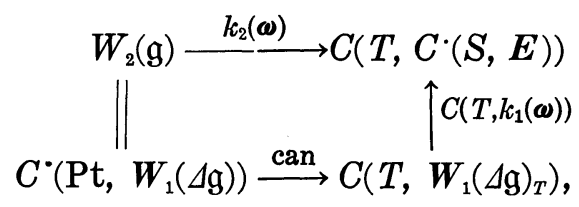

where the coefficient map $k_{1}(\omega): W_{1}(\Delta \mathrm{g})_{T} \rightarrow C^{*}(S, E)$ is determined for every simplex $\tau=\left(k_{0}, \cdots, k_{m}\right) \in T_{m}$ as follows. Associated to $\tau$ is the $(m+1)$-tuple of families of connections $\boldsymbol{\omega}_{\tau}=\left(\boldsymbol{\omega}_{k_{0}}, \cdots, \boldsymbol{\omega}_{k_{m}}\right)$ on $\boldsymbol{E}$. As in (3.30) this determines morphisms of $\mathrm{g}$-DG-algebras

$$
k_{1}\left(\boldsymbol{\omega}_{\tau}\right): W_{1}\left(\mathrm{~g}^{m+1}\right) \rightarrow C^{\cdot}(S, \boldsymbol{E}), \quad m \geqq 0 .
$$

The coefficient map $k_{1}(\omega)$ is now defined at $\tau \in T_{m}$ by $k_{1}\left(\omega_{\tau}\right)$. Explicitly, for $\sigma \in S_{l}, l \geqq 0$, the map

$$
k_{1}\left(\omega_{\tau}\right)_{o}: W_{1}^{l}\left(\mathfrak{g}^{m+1}\right)=\bigotimes_{0}^{m} \bigotimes_{0}^{l} W(\mathrm{~g}) \rightarrow E_{o}
$$

is given by the matrix $\left(\omega_{k_{\alpha}, j_{\beta}}\right) \alpha=0, \cdots, m ; \beta=0, \cdots, l$ of $\mathrm{g}$-connections.

A vertex $\alpha \in S_{0}$ is called conical if the mapping $\sigma=\left(i_{0}, \cdots, i_{l}\right) \mapsto$ $\sigma_{\alpha}=\left(\alpha, i_{0}, \cdots, i_{l}\right)$ maps $S_{l}$ into $S_{l+1}$ for all $l \geqq 0$. It is well-known (see e.g., [7, Ch. I, 3.7]) that the existence of a conical vertex implies the acyclicity of the cochain complex $C \cdot(S, A)$ for any abelian group $A$. More precisely, the mappings $\mathscr{H}_{l}: C^{l}(S, A) \rightarrow C^{l-1}(S, A)$ defined for $l>0$ by the formula $\mathscr{H}_{l}(c) \sigma=c\left(\sigma_{\alpha}\right)$ for $\sigma \in S_{l-1}, c \in C^{l}$ satisfy the homotopy relations

$$
\delta_{l-1} \circ \mathscr{H}_{l}+\mathscr{H}_{l+1} \circ \delta_{l}=\mathrm{id}_{c l}, \quad l>0
$$

and

$$
\mathscr{H}_{1} \circ \delta_{0}=\operatorname{id}_{C^{0}}-\varepsilon \circ j_{\alpha}, \quad j_{\alpha^{\circ}} \varepsilon=\mathrm{id}_{A} .
$$

Here $A \underset{j_{\alpha}}{\stackrel{\varepsilon}{\rightleftarrows}} C^{\circ}(S, A)$ denote the mapping of $A$ to constant 0-cochains and evaluation of 0 -cochains at $\alpha \in S_{0}$ respectively. It follows that the augumented complex

$$
A \underset{j_{\alpha}}{\stackrel{\varepsilon}{\longrightarrow}} C^{0}(S, A) \stackrel{\delta_{0}}{\rightarrow} C^{1}(S, A) \stackrel{\delta_{1}}{\rightarrow} \cdots
$$


is acyclic, i.e.

$$
H_{\delta}^{l}(C(S, A)) \stackrel{\varepsilon}{\cong}\left\{\begin{array}{l}
0, l>0 \\
A, l=0
\end{array}\right.
$$

with the isomorphism in degree 0 induced by $j_{\alpha}$ and $\varepsilon$. If $A$ is now a DG-algebra, $C \cdot\left(S, A^{*}\right)$ is a double complex (see Section 2), and (3.37) implies that one of its spectral sequences collapses. Hence we have the following fact.

Proposition 3.38. Let $S$ be a simplicial set with a conical vertex $\alpha$ and let $A^{\cdot}$ be a DG-algebra. Then there is a canonical isomorphism (induced by $\varepsilon$ )

$$
\varepsilon_{*}: H^{\cdot}(A) \stackrel{\cong}{\rightarrow} H^{\cdot}\left(S ; A^{\bullet}\right) \equiv H\left(C^{t \circ t}(S ; A)\right) \text {. }
$$

Furthermore $j_{\alpha^{*}}=\varepsilon_{*}^{-1}$ and thus $j_{\alpha^{*}}$ is independent of the choice of the conical vertex $\alpha \in S_{0}$.

Proposition 3.38 applies in particular to the standard ss set $\Delta_{(p)}$ of singular simplices in the set $\Delta_{p}=\{0, \cdots, p\}, p \geqq 0$, in which every vertex is conical.

We turn now to the canonical projections $\rho_{s}: W_{s} \rightarrow W_{s-1}$ (see 2.24) for $s=1,2$. For any vertex $k \in T_{0}$ we have by the construction of $k_{2}(\omega)$ in (3.33) a commutative diagram

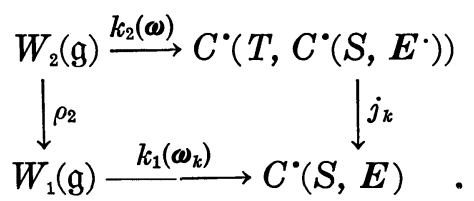

Similarly for a family of connections $\omega=\left(\omega_{j}\right)_{j \in S_{0}}$ in $E$ and a vertex $k \in T_{0}$ we have a commutative diagram

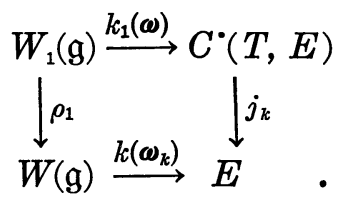

For conical vertices $k \in T_{0}$, the vertical maps on the RHS in 3.39, resp. 3.40 are by Proposition 3.38 isomorphisms in cohomology, which do not depend on $k$. Together with the results of Sections 4 and 5 this will imply the connection-independence of the generalized characteristic homomorphism of a foliated bundle (see Section 6, in particular Propositon 6.11). 
The construction of $\lambda_{E}^{l}$ shows that for a constant local system on $S$ given by a g-DG-algebra $E$ (see Section 2), and a family $\omega=\left(\omega_{j}\right)_{j \in S_{0}}$ of connections in $E$, we have

$$
\lambda_{E}^{l}(\sigma)=\lambda_{E}^{l}(\sigma): W(\mathrm{~g})^{\cdot} \rightarrow E_{\sigma}^{\bullet^{-l}}=E^{\cdot-l} .
$$

Thus the mappings $\lambda_{E}^{l}(\sigma)$ introduced before are a special case of the mappings $\lambda_{E}^{l}(\sigma)$. In particular for $S=\mathrm{Pt}$ and a single connection $\omega$ in $E$ we obtain for $\sigma_{l}=(0, \cdots, 0) \in \mathrm{Pt}_{l}$

$$
\lambda_{E}^{l}\left(\sigma_{l}\right): W(\mathrm{~g}) \rightarrow C^{l}(\mathrm{Pt}, E) \cong E,
$$

where $\lambda_{E}^{l}\left(\sigma_{l}\right)=\lambda_{E}^{l}(\omega, \cdots, \omega)=(k(\omega), \cdots, k(\omega)) \circ \lambda^{l}$. In this situation we have the following facts.

Proposition 3.41.

(i) For any g-DG-algebra $E$ and connection $\omega$ in $E$ we have

$$
\lambda_{E}^{l}(\omega, \cdots, \omega)=\lambda_{E}^{l}\left(\sigma_{l}\right)=0 \text { for } l>0 ;
$$

(ii) $\mu \circ \lambda^{l}=0, l>0$, where $\mu=\mu_{0}^{0} \circ \cdots \circ \mu_{l-1}^{l-1}: W(\mathfrak{g})^{\otimes l+1} \rightarrow W(\mathfrak{g})$ denotes l-fold multiplication in $W(\mathfrak{g})$.

Proof. Since $k(\omega)$ is multiplicative we have by $\left(3.28^{\prime}\right)$ a commutative diagram

$$
\begin{aligned}
& W(\mathfrak{g}) \stackrel{\lambda^{l}}{\longrightarrow} W_{1}^{l}(\mathfrak{g})=W(\mathfrak{g})^{\otimes l+1} \stackrel{\mu}{\longrightarrow} W(\mathfrak{g}) \\
& \mid k(\omega) \otimes \cdots \otimes k(\omega) \backslash \searrow^{k\left(\omega_{\sigma_{l}}\right)} \downarrow k(\omega) \\
& E \otimes \cdots \otimes E \stackrel{\mu_{E}}{\longrightarrow} C^{\imath}(\mathrm{Pt}, E) \cong E .
\end{aligned}
$$

The equivalence of the two statements is now clear from (3.32). To prove (i), we observe that by the definitions (3.9), (3.10) we have $\lambda_{E}^{l}\left(\sigma_{l}\right)=$ $\pi_{*}^{l} \circ k\left(\omega^{o}\right)$ and $\omega^{o} l=\sum_{j=0}^{l} t_{j} \omega=\omega$. Hence $k\left(\omega^{\sigma}\right)$ is independent of $t_{j}$ and $d t_{j}, j=0, \cdots, l$ and therefore $\pi_{*}^{(l)} \circ k\left(\omega^{\sigma} l\right)=0$ by Definition (3.4).

Proposition 3.41 says that the difference maps $\lambda_{E}^{l}, l>0$ for a constant family of connections are all zero. Thus if $S$ is a ss set and $\omega=$ $\left(\omega_{j}\right)_{j \in S_{0}}$ a constant family of connections in $E$ given by $\omega_{j}=\omega, j \in S_{0}$, then the diagram

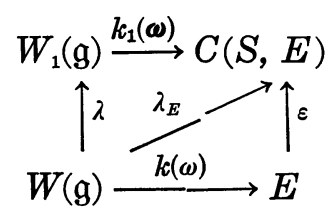

is commutative. Again $\varepsilon$ induces an isomorphism in cohomology if $S$ 
admits a conical vertex (e.g., in the standard ss set $\Delta_{(p)}, p \geqq 0$, where every vertex is conical).

4. Filtrations. Recall the canonical (Koszul) filtration on a $g$-DGalgebra $E \cdot$ defined by

$$
F^{p} E^{n}=\left\{a \in E^{n} \mid v . a=0 \text { for } v \in V_{q} \text { with } q>n-p\right\} .
$$

This property is equivalently expressed by

$$
v . a=0 \text { for } v \in \wedge_{q} g \text { with } q>n-p
$$

and still equivalently by

$$
v . a=0 \text { for } v=x_{1} \wedge \cdots \wedge x_{n-p+1}, \quad x_{i} \in \mathfrak{g} .
$$

The following properties of the canonical filtration are important:

$$
\begin{gathered}
F^{p} E \supset F^{p+1} E, F^{0} E=E \text { and } F^{p} E^{n}=0 \text { for } p>n ; \\
F^{p} E \subset E \text { is a g-DG-ideal ; } \\
F^{p} E \cdot F^{q} E \subset F^{p+q} E ; \\
F^{p} E^{p}=\left(E^{i(8)}\right)^{p}, \text { i.e., the elements } a \text { of degree } p \\
\text { such that } i(x) a=0 \text { for all } x \in \mathfrak{g} .
\end{gathered}
$$

A homomorphism (of degree zero) of g-DG-algebras $E^{\cdot} \rightarrow E^{\prime \prime}$ is clearly filtration preserving. More generally a homomorphism $E \cdot \rightarrow E^{\prime \cdot-l}$ of degree $-l$ sends $F^{p} E^{n}$ into $F^{p-l} E^{\prime n-l}$. If we want to emphasize the dependence of the filtration on $\mathfrak{g}$, we write $F^{p} E=F^{p}(\mathfrak{g}) E$. For the Weil algebra $W(\mathfrak{g})$ e.g.,

$$
F^{2 p-1}(\mathfrak{g}) W(\mathfrak{g})=F^{2 p}(\mathfrak{g}) W(\mathfrak{g})=S^{p} \mathfrak{g}^{*} . W(\mathfrak{g}), \quad p \geqq 0 .
$$

For the Weil algebra $W(L)$ of a Lie algebra $L$ over $\mathfrak{g}$ we have more generally

$$
F^{p}(\mathfrak{g}) W(L)=\prod_{s+2 t \geqq p} \wedge^{s} V(L, \mathfrak{g})^{*} . \wedge L^{*} \otimes S^{t} L^{*}, \quad p \geqq 0 .
$$

Here $V(L, \mathfrak{g})$ is defined by the short exact sequence

$$
0 \rightarrow \mathfrak{g} \rightarrow L \rightarrow V(L, \mathfrak{g}) \rightarrow 0,
$$

so that $V(L, \mathfrak{g})^{*} \subset L^{*}$.

The universal property (3.7) of the Weil algebra is a consequence of the following refined result, which is essential for the considerations of this paper.

Proposition 4.7. Let $L$ be a Lie algebra over $\mathfrak{g}, \mathfrak{h} \subset \mathfrak{g}$ any subalgebra and $E^{\cdot}$ a commutative g-DG-algebra. Let $\omega: L^{*} \rightarrow E^{1}$ be a linear 
map such that

$$
\begin{aligned}
& i(x) \omega(\alpha)=i(x) \alpha .1 \text { for } x \in \mathfrak{g}, \quad \alpha \in L^{*} ; \\
& \theta(y) \omega(\alpha)=\omega(\theta(y) \alpha) \text { for } y \in \mathfrak{h}, \quad \alpha \in L^{*} .
\end{aligned}
$$

(i ) There exists a unique homomorphism of DG-algebras

$$
k(\omega): W(L) \rightarrow E
$$

(the Weil homomorphism of $\omega$ ), making the diagram

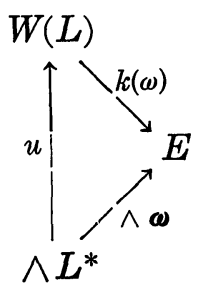

commutative, where $u: \wedge L^{*} \rightarrow W(L)=\wedge L^{*} \otimes S L^{*}$ denotes the universal connection $a \rightarrow a \otimes 1$ and $\wedge \omega$ the multiplicative extension of $\omega$ (this map will again be denoted $\omega$ as already done before).

(ii) $k(\omega)$ is an h-DG-homomorphism.

(iii) For the g-filtration $F(\mathfrak{g}) E \cdot$ we have the property

$$
k(\omega): \wedge^{s} V(L, \mathfrak{g})^{*} . \wedge L^{*} \otimes S^{t} L^{*} \rightarrow F^{s+t}(\mathfrak{g}) E
$$

REMARK 4.13. Note that if (4.9) is replaced by the same condition for all $x \in \mathfrak{g}$, then $\omega=\wedge \omega: \wedge^{\bullet} L^{*} \rightarrow E^{*}$ is a $V(\mathfrak{g})$-algebra homomorphism, and so is then $k(\omega)$. Therefore

$$
k(\omega): F^{p}(\mathfrak{g}) W(L)^{\bullet} \rightarrow F^{p}(\mathfrak{g}) E^{\bullet}, \quad p \geqq 0 .
$$

But in the more general situation discussed in Proposition 4.7, the subspace

$$
\wedge^{8} V(L, \mathfrak{g})^{*} \otimes S^{t} L^{*} \subset F^{8+2 t}(\mathfrak{g}) W(L)
$$

is only mapped into $F^{s+t}(\mathfrak{g}) E^{*}$, so that $k(\omega)$ does not preserve the canonical $\mathrm{g}$-filtrations.

Proof. First $\omega$ is extended to a multiplicative homomorphism $\omega=$ $\wedge \omega: \wedge^{*} L^{*} \rightarrow E^{*}$. This defines $k(\omega)$ on $L^{*} \otimes 1 \subset W(L)$. The multiplicative map $k(\omega)$ on $1 \otimes S L^{*}$ is characterized by its effect on elements $1 \otimes$ $\tilde{\alpha} \in 1 \otimes S^{1} L^{*}$, where as usual $\tilde{\alpha} \in S^{1} L^{*}$ corresponds to $\alpha \in \wedge^{1} L^{*}$. By definition

$$
k(\omega) \tilde{\alpha}=d_{E} \omega(\alpha)-\omega\left(d_{\wedge} \alpha\right)
$$

where $d_{\wedge}$ denotes the Chevalley-Eilenberg differential in $\wedge L^{*}$. First 
we have to verify that the algebra homomorphism $k(\omega)$ commutes with the differentials. The formula

$$
k(\omega) d_{W}=d_{E} k(\omega)
$$

is verified on generators exactly as in [16], p. 58. To prove the formula

$$
k(\omega) \theta(y)=\theta(y) k(\omega) \text { for } y \in \mathfrak{h},
$$

we first observe that on $\wedge L^{*}$ it is true by assumption. For $\tilde{\alpha} \in S^{1} L^{*}$ we have then

$$
\begin{aligned}
(k(\omega) \theta(y))(\tilde{\alpha}) & =\left(d_{E} \omega-\wedge \omega \circ d_{\wedge}\right)(\theta(y) \alpha)=d_{E} \theta(y) \omega(\alpha)-\wedge \omega \circ \theta(y) d_{\wedge} \alpha \\
& =\theta(y)\left(d_{E} \omega(\alpha)-\wedge \omega d_{\wedge} \alpha\right)=\theta(y) k(\omega) \tilde{\alpha}
\end{aligned}
$$

which verifies (4.15). The formula

$$
k(\omega) i(y)=i(y) k(\omega) \text { for } y \in \mathfrak{h}
$$

is again true on $\wedge L^{*}$ by the assumption 4.8. For $\tilde{\alpha} \in S^{1} L^{*}$ we have on the one hand $k(\omega) i(y) \widetilde{a}=0$ (since the derivations $i(y)$ are zero on $\left.S \cdot L^{*}\right)$. On the other hand for any $y \in \mathfrak{g}$

$$
\begin{aligned}
i(y) k(\omega) \widetilde{\alpha} & =i(y)\left(d_{E} \omega(\alpha)-\wedge \omega d \alpha\right) \\
& =\left(\theta(y)-d_{E} i(y)\right) \omega(\alpha)-\wedge \omega\left(\theta(y)-d_{\wedge} i(y)\right) \alpha \\
& =(\theta(y) \circ \omega-\omega \circ \theta(y)) \alpha .
\end{aligned}
$$

Here we used $\left(d_{E} i(y)\right) \omega(\alpha)=d_{E} i(y) \alpha=0$ and $d_{\wedge} i(y) \alpha=0$. Assumption (4.9) implies that this term vanishes for $y \in \mathfrak{h}$ and hence $i(y) k(\omega) \tilde{\alpha}=0$. This finishes the proof of part (ii).

It remains to prove (4.12). By the multiplicativity of $k(\omega)$ and the filtration $F^{p}(\mathfrak{g}) E$, it suffices to check the following facts:

$$
\begin{aligned}
& k(\omega) \alpha \in F^{1}(\mathrm{~g}) E^{1} \quad \text { for all } \alpha \in V(L, \mathfrak{g})^{*} \subset L^{*} \text {; } \\
& k(\omega) \tilde{\alpha} \in F^{1}(\mathfrak{g}) E^{2} \quad \text { for all } \quad \tilde{\alpha} \in S^{1} L^{*} \text {. }
\end{aligned}
$$

But (4.18) means by (4.5) that

$$
i(x) k(\omega) \alpha=0 \text { for } x \in \mathfrak{g} \text { and } \alpha \in L^{*} \text { with } \alpha \mid \mathfrak{g}=0 \text {. }
$$

This is clear since in fact with these assumptions

$$
i(x) k(\omega) \alpha=i(x) \omega(\alpha)=i(x) \alpha=0 .
$$

Property (4.19) means by (4.1) that

$$
i(x) i(y) k(\omega) \tilde{\alpha}=0 \text { for } x, y \in \mathfrak{g} \text { and } \tilde{\alpha} \in S^{1} L^{*} .
$$

Now by (4.17) we have for any $y \in \mathfrak{g}$ the identity $i(y) k(\omega) \alpha=(\theta(y) \circ \omega-$ $\omega \circ \theta(y)) \alpha$ and therefore 


$$
i(x) i(y) k(\omega) \tilde{\alpha}=i(x) \theta(y) \omega(\alpha)-\omega(i(x) \theta(y) \alpha) .
$$

Using the identity $\theta(y) i(x)-i(x) \theta(y)=i[y, x]$ (see (2.9)), it follows that

$$
\begin{aligned}
i(x) i(y) k(\omega) \tilde{\alpha} & =(\theta(y) i(x)+i[x, y]) \omega(\alpha)-\omega((\theta(y) i(x)+i[x, y]) \alpha) \\
& =\theta(y)(i(x) \omega(\alpha)-\omega(\theta(y) i(x) \alpha) .
\end{aligned}
$$

The last simplification follows from the assumption (4.8). But clearly $\theta(y)(i(x) \omega(\alpha)=\theta(y) \alpha(x) .1=0$ and similarly for the second term, which establishes (4.20). This finishes the proof of Proposition 4.7.

As an application of Proposition 4.7 we note that the Weil algebra functor $W$ on the category $\mathscr{L}_{8}$ of Lie algebras $L$ over a fixed Lie algebra $g$ extends to linear maps $\varphi: L^{\prime} \rightarrow L$ making the diagram

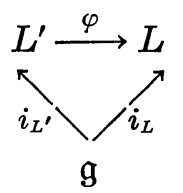

commutative. The composition $L^{*} \stackrel{\varphi^{*}}{\rightarrow} L^{\prime *} \subset W\left(L^{\prime}\right)$ extends by part (i) of Proposition 4.7 to a unique homomorphism of DG-algebras

$$
k(\varphi): W(L) \rightarrow W\left(L^{\prime}\right) \text {. }
$$

The multiplicative map $k(\varphi)$ is characterized by its values on generators $\alpha \in \wedge^{1} L^{*}, \tilde{\alpha} \in S^{1} L^{*}$. For $\alpha \in \wedge^{1} L^{*}$ we have clearly

$$
k(\varphi) \alpha=\varphi^{*} \alpha \otimes 1 \text {. }
$$

For $\tilde{\alpha} \in S^{1} L^{*}$ we have by (4.14) (again we identify $\phi^{*}$ with its multiplicative extensions to $\wedge L^{*}$ )

$$
\begin{aligned}
k(\varphi) \tilde{\alpha} & =d_{W}\left(\varphi^{*} \alpha \otimes 1\right)-\phi^{*}\left(d_{\wedge} \alpha\right) \otimes 1 \\
& =1 \otimes d^{\prime \prime} \phi^{*} \alpha+\left(d_{\wedge} \varphi^{*} \alpha-\varphi^{*}\left(d_{\wedge} \alpha\right)\right) \otimes 1 \\
& =1 \otimes \widetilde{\varphi^{*} \alpha}+\alpha K(\varphi) \otimes 1
\end{aligned}
$$

where

$$
\alpha K(\varphi)=d_{\wedge} \varphi^{*} \alpha+\frac{1}{2} \alpha[\varphi, \varphi] .
$$

Here we have used the identity (see e.g., [16], (3.6))

$$
\frac{1}{2} \alpha[\varphi, \varphi]=-\varphi^{*}\left(d_{\wedge} \alpha\right) \text {. }
$$

The evaluation of $\alpha K(\phi) \in \wedge^{2} L^{\prime *}$ for $x, y \in L^{\prime}$ gives 


$$
\alpha K(\varphi)(x, y)=-\varphi^{*} \alpha[x, y]+\alpha[\varphi x, \phi y]=\alpha([\varphi x, \phi y]-\phi[x, y])
$$

so that $K(\varphi)$ measures the deviation of $\varphi$ from being a Lie homomorphism. It follows that for a Lie homomorphism $\phi$ the extension $k(\varphi)$ on $1 \otimes S L^{*}$ is simply the multiplicative extension of $\phi^{*}: L^{*} \rightarrow L^{\prime *}$. Thus for a Lie homomorphism $\varphi: L^{\prime} \rightarrow L$ (over g) the map $k(\varphi)$ coincides with the functorial map $W(\varphi)$.

For any linear map $\varphi: L^{\prime} \rightarrow L$ (over g) condition (4.8) holds and by (4.18) (4.19) we have

$$
\begin{aligned}
& k(\varphi) \alpha \in \wedge^{1} V\left(L^{\prime}, \mathfrak{g}\right)^{*} \text { for } \alpha \in V(L, \mathfrak{g})^{*} \\
& k(\varphi) \tilde{\alpha} \in\left(\wedge^{1} V\left(L^{\prime}, \mathfrak{g}\right)^{*} . \wedge^{1} L^{\prime *}\right) \otimes \Gamma \Perp \Gamma \otimes S^{1} L^{*} \quad \text { for } \quad \tilde{\alpha} \in S^{1} L^{*},
\end{aligned}
$$

where $\Gamma$ stands for the ground field. By multiplicativity it follows that

(4.26) $\quad k(\varphi): \wedge^{s} V(L, \mathfrak{g})^{*} \otimes S^{t} L^{*} \rightarrow \Perp_{u+v=t} \wedge^{s+u} V\left(L^{\prime}, \mathfrak{g}\right)^{*} . \wedge L^{*} \otimes S^{v} L^{\prime *}$,

and with $q=\operatorname{dim} L^{\prime} / \mathfrak{g}=\operatorname{dim} V\left(L^{\prime}, \mathfrak{g}\right)$ clearly $s+u \leqq q$. Thus $u \leqq$ $\min (t, q-s)$ and

$$
\begin{aligned}
s+u+2 v & =s+2(u+v)-u=s+2 t-u \\
& \geqq s+2 t-\min (t, q-s) .
\end{aligned}
$$

It follows that

$$
\begin{aligned}
k(\varphi): & \wedge^{s} V(L, \mathfrak{g})^{*} \otimes S^{t} L^{*} \rightarrow F^{s+2 t-\min (t, q-s)}(\mathfrak{g}) W\left(L^{\prime}\right) \\
& F^{s+2 t}(\mathfrak{g}) W(L) .
\end{aligned}
$$

It is convenient to introduce on the functor $W(-)$ on $\mathscr{L}_{\mathbb{B}}$ the following even filtration (as distinguished from the canonical filtration (4.6))

$$
F_{0}^{2 p}(\mathfrak{g}) W(L)=\mathscr{J}^{p},
$$

where $\mathscr{J}=\left(W(L)^{i(8)}\right)^{+} . W(L)$. This reads explicitly as follows

$$
F_{0}^{2 p}(\mathfrak{g}) W(L)=\underset{s+t \geqq p}{\Perp} \wedge^{s} V(L, \mathfrak{g})^{*} . \wedge L^{*} \otimes S^{t} L^{*}, \quad p \geqq 0,
$$

$F_{0}^{*}(\mathrm{~g})$ is a decreasing, multiplicative filtration by $\mathrm{g}$-DG-ideals in $W(L)$. The fact that $F_{0}^{2 p}(\mathrm{~g}) W(L)$ is invariant under the operations $i(x), \theta(x)$ for $x \in \mathfrak{g}$ is immediate from the definition of $F_{0}(\mathrm{~g})$. The ideal $F_{0}^{2 p}(\mathrm{~g}) W(L)$ is invariant under the Weil differential $d_{W}=d^{\prime}+d^{\prime \prime}$ by definition of $d_{W}$ and the fact that $d^{\prime} \alpha=d_{\wedge} \alpha$ satisfies $i(y) i(x) d_{\wedge} \alpha=-\alpha[x, y]=0$ for $x, y \in \mathfrak{g}$, $\alpha \in V(L, \mathfrak{g})^{*}$, and hence $d_{\wedge} \alpha \in\left(\wedge V(L, \mathfrak{g})^{*} \cdot \wedge L^{*}\right)^{2}$.

From (4.6) and (4.30) it follows

$$
F_{0}^{2 p}(\mathfrak{g}) W(L) \subset F^{p}(\mathfrak{g}) W(L), \quad p \geqq 0 .
$$


Using $F_{0}(\mathfrak{g})$ we can now rephrase the filtration properties of $k(\omega)$ in (4.12) and of $k(\varphi)$ in (4.26) as follows.

CoRollary 4.32. (i) Let $\omega: L^{*} \rightarrow E^{1}$ be given as in Proposition 4.7. Then

$$
k(\omega): F_{0}^{2 p} W(L) \rightarrow F^{p}(\mathfrak{g}) E \text { for all } p \geqq 0,
$$

i.e., $k(\omega)$ preserves the filtrations $F_{0}^{*}(\mathrm{~g})$ and $F^{*}(\mathrm{~g})$ (up to the factor 2).

(ii) Let $\varphi: L^{\prime} \rightarrow L$ be a linear map over $\mathfrak{g}$, where $L, L^{\prime}$ lare Lie algebras over g. Then

$$
k(\varphi): F_{0}^{2 p}(\mathfrak{g}) W(L) \rightarrow F_{0}^{2 p}(\mathfrak{g}) W\left(L^{\prime}\right) \text { for all } p \geqq 0,
$$

i.e., the filtration $F_{0}^{*}(\mathrm{~g})$ is defined on the functor $W: \mathscr{L}_{\mathrm{g}} \rightarrow \mathscr{A}_{\mathrm{g}}$ and on its extension to linear maps over $\mathrm{g}$.

All this applies in particular to the situation where $\phi=\theta: L \rightarrow \mathfrak{g}$ is a retraction of the inclusion $i=i_{L}: \mathrm{g} \rightarrow L$, i.e., $\theta \circ i=\mathrm{id}$. Then there is a unique DG-homomorphism

$$
k(\theta): W(\mathfrak{g}) \rightarrow W(L)
$$

extending $\theta^{*}$. It is an $\mathfrak{h}$-DG-homomorphism for any subalgebra $\mathfrak{h} \subset \mathfrak{g}$ with respect to which $\theta$ is h-equivariant. This follows from part (ii) of Proposition 4.7. The property

$$
k(\theta): F_{0}^{2 p}(\mathrm{~g}) W(\mathrm{~g}) \rightarrow F_{0}^{2 p}(\mathrm{~g}) W(L)
$$

will be used below. Note that for $L=\mathfrak{g}$ the quotient $V(L, \mathfrak{g})=0$, so that $F_{0}^{2 p}(\mathrm{~g}) W(\mathrm{~g})=F^{2 p}(\mathrm{~g}) W(\mathrm{~g})$. (4.28) implies then in contrast to (4.34)

$$
k(\theta): F^{2 p}(\mathfrak{g}) W(\mathfrak{g}) \rightarrow F^{2 p-\min (p, q)}(\mathfrak{g}) W(L)
$$

with $q=\operatorname{dim} L / g$ (this property was used in [18] and appears there as part (ii) of Lemma 4.9).

We have just discussed the properties of the unique extension of $L^{*} \stackrel{\varphi^{*}}{\rightarrow} L^{\prime *} \subset W\left(L^{\prime}\right)$ to the DG-homomorphism (4.22). But a linear map $\varphi: L^{\prime} \rightarrow L$ (over g) leads also more simply to the composition $L^{*} \rightarrow L^{\prime *} \subset$ $\wedge L^{\prime *}$. Its unique extension to a DG-homomorphism

$$
\Delta(\varphi): W(L) \rightarrow \wedge L^{\prime *}
$$

is of course the composition

$$
\Delta(\varphi)=\pi \circ k(\varphi)
$$

where $\pi: W\left(L^{\prime}\right) \rightarrow \wedge L^{\prime *}$ denotes the canonical projection. By (4.23) (4.24) it follows that $\Delta(\varphi)$ is characterized by the formulas 


$$
\left\{\begin{array}{l}
\Delta(\varphi) \alpha=\varphi^{*} \alpha \\
\Delta(\varphi) \tilde{\alpha}=\alpha K(\varphi)
\end{array}\right.
$$

for $\alpha \in \wedge{ }^{1} L^{*}, \tilde{\alpha} \in S^{1} L^{*}$.

On $\wedge L^{*}, L \in \mathscr{L}_{\mathrm{g}}$ we may consider the filtration $F_{0}^{*}(\mathfrak{g})$ induced by the canonical projection $\pi: W(L) \rightarrow \wedge L^{*}$. Since there are no symmetric elements in $\wedge L^{*}$, it follows from (4.6), (4.30) that

$$
F_{0}^{2 p}(\mathfrak{g}) \wedge L^{*}=F^{p}(\mathfrak{g}) \wedge L^{*} \text { for } p \geqq 0,
$$

i.e., the $g$-filtration $F_{0}^{2 p}(\mathfrak{g})$ coincides on $\wedge L^{*}$ with the canonical $g$-filtration $F^{p}(\mathfrak{g})$.

By (4.36) and Corollary 4.32, (ii) we have for $\Delta(\varphi), \varphi: L \rightarrow L^{\prime}$ a linear map over $\mathfrak{g}$ :

$$
\Delta(\varphi): F_{0}^{2 p}(\mathfrak{g}) W(L) \rightarrow F_{0}^{2 p}(\mathfrak{g}) \wedge L^{\prime *}=F^{p}(\mathfrak{g}) \wedge L^{\prime *} \text { for } \quad p \geqq 0 .
$$

We turn now to the filtration properties for the $V(L)$ - resp. $V(\mathfrak{g})$ module map

$$
\lambda^{l}: W(L)^{\bullet} \rightarrow W\left(L^{l+1}\right)^{\bullet-l}
$$

constructed in Section 3 (see Theorem 3.26). Clearly

$$
\lambda^{l}: F^{p}(\mathfrak{g}) W(L)^{\cdot} \rightarrow F^{p-l}(\mathfrak{g}) W\left(L^{l+1}\right)^{\cdot-l} \text { for } p \geqq l
$$

as is the case for any $g$-DG-map of degree $-l$. Concerning the filtration $F_{0}(\mathfrak{g})$, we have the following fact.

Proposition 4.41.

$$
\lambda^{l}: F_{0}^{2 p}(\mathfrak{g}) W(L)^{\bullet} \rightarrow F_{0}^{2 p}(\mathfrak{g}) W\left(L^{l+1}\right)^{\bullet-l}, \quad p \geqq 0 .
$$

Proof. First we make explicit the filtration $F_{0}(\mathfrak{g})$ on $W\left(L^{l+1}\right)$. Let $V_{l}(L, \mathfrak{g})$ be defined by the exact sequence

$$
0 \rightarrow \mathfrak{g} \stackrel{\Delta \circ i}{\rightarrow} L^{l+1} \rightarrow V_{l}(L, \mathfrak{g}) \rightarrow 0
$$

Thus $V_{0}(L, \mathfrak{g})=V(L, \mathfrak{g})$ in the notation of (4.30). Then

$$
F_{0}^{2 p}(\mathfrak{g}) W\left(L^{l+1}\right)=\underset{s+t \geqq p}{\Perp} \wedge^{s} V_{l}(L, \mathfrak{g})^{*} . \wedge L^{* l+1} \otimes S^{t} L^{* l+1}, \quad p \geqq 0 .
$$

Let $s+t=p$ and consider an element

$$
w=v \cdot \alpha_{1} \wedge \cdots \wedge \alpha_{s} \otimes \widetilde{\beta}_{1} \cdots \widetilde{\beta}_{t} \in \wedge^{s} V(L, \mathfrak{g})^{*} \wedge L^{*} \otimes S^{t} L^{*},
$$

where $\alpha_{i} \in \wedge^{1} V(L, \mathfrak{g})^{*}$ and $\widetilde{\beta}_{j} \in S^{1} L^{*}, i=1, \cdots, s, j=1, \cdots, t$. We have to show $\lambda^{l}(w) \in F_{0}^{2 p} W\left(L^{l+1}\right)$. Since by $(3.26)$ we have $\lambda^{l}(w)=0$ for $l>t$, we can assume that $l \leqq t$. Recall from Section 3 that by definition $\lambda^{l}$ is the composition 


$$
\lambda^{l}: W(L) \stackrel{k\left(u^{\sigma}\right)}{\longrightarrow} W\left(L^{l+1}\right)^{(l)} \stackrel{\pi_{*}^{(l)}}{\longrightarrow} W\left(L^{l+1}\right), \quad \sigma=\sigma_{l} \in \mathrm{Pt}_{l}
$$

where $u_{j}: \wedge(L)^{*} \rightarrow W\left(L^{l+1}\right) \cong W(L)^{\otimes l+1}$ are the canonical connections given by inclusion into the $j^{\text {th }}$ factor, $j=0, \cdots, l$, and $u^{\sigma}=\sum_{j=0}^{l} t_{j} u_{j}$ with $\sum_{j=0}^{l} t_{j}=1$. As $k\left(u^{\sigma}\right)$ is multiplicative, we have

$$
k\left(u^{\sigma}\right) w=k\left(u^{\sigma}\right) v \cdot k\left(u^{\sigma}\right) \alpha_{1} \cdots k\left(u^{\sigma}\right) \alpha_{s} \cdot k\left(u^{\sigma}\right) \widetilde{\beta}_{1} \cdots k\left(u^{\sigma}\right) \widetilde{\beta}_{t} \in W\left(L^{l+1}\right)^{l} .
$$

We will show below that all the coefficients of the factors $k\left(u^{\sigma}\right) \alpha_{i}$ and $k\left(u^{\sigma}\right) \widetilde{\beta}_{j}$ are elements of $F_{0}^{2}(\mathrm{~g}) W\left(L^{l+1}\right)$. It follows by the multiplicativity of $F_{0}^{*}(\mathrm{~g})$ that the coefficients of the terms containing $d t_{1} \wedge \cdots \wedge d t_{l}$ must be in $F_{0}^{2 p}(\mathrm{~g}) W\left(L^{l+1}\right), p=s+t$, and hence $\pi_{*}^{(l)}\left(k\left(u^{\sigma}\right) w\right) \in F_{0}^{2 p}(\mathfrak{g}) W\left(L^{l+1}\right)$ by the Definition (3.4) of $\pi_{*}^{(l)}$. It remains therefore to verify the formulas

$$
\begin{array}{lll}
k\left(u^{\sigma}\right) \alpha \in F_{0}^{2}(\mathfrak{g}) W\left(L^{l+1}\right) & \text { for } & \alpha \in \wedge^{1} V(L, \mathfrak{g})^{*} \\
k\left(u^{\sigma}\right) \widetilde{\beta} \in F_{0}^{2}(\mathfrak{g}) W\left(L^{l+1}\right) & \text { for } & \widetilde{\beta} \in S^{1} L^{*}
\end{array}
$$

For conciseness we introduce the notation

$$
\left\{\begin{array}{l}
\alpha^{(j)}=k\left(u_{j}\right)(\alpha)=1 \otimes \cdots \otimes \alpha \otimes \cdots \otimes 1(\alpha \text { in } j \text {-th place }) \text { for } \alpha \in \wedge^{1} L^{*} \\
\widetilde{\beta}^{(j)}=k\left(u_{j}\right) \widetilde{\beta}=1 \otimes \cdots \otimes \widetilde{\beta} \otimes \cdots \otimes 1(\widetilde{\beta} \text { in } j \text {-th place }) \text { for } \widetilde{\beta} \in S^{1} L^{*} .
\end{array}\right.
$$

Note that $V_{l}(L, \mathfrak{g})^{*}$ in $(4.42)$ is determined by an obvious split exact sequence

and that

$$
0 \longrightarrow V_{l}(L, L)^{*} \longrightarrow V_{l}(L, \mathfrak{g})^{*} \longrightarrow V(L, \mathfrak{g})^{*} \longrightarrow 0
$$

$$
\begin{aligned}
& \alpha^{(j)}-\alpha^{(k)} \in V_{l}(L, L)^{*} \text { for } \alpha \in \wedge^{1} L^{*}, \\
& \alpha^{(j)} \in V_{l}(L, \mathfrak{g})^{*} \text { for } \alpha \in V(L, \mathfrak{g})^{*} .
\end{aligned}
$$

We have then directly from the definition of $u^{\sigma}$

$$
k\left(u^{\sigma}\right) \alpha=\sum_{j=0}^{l} t_{j} \alpha^{(j)}=\alpha^{(0)}+\sum_{j=1}^{l} t_{j}\left(\alpha^{(j)}-\alpha^{(0)}\right)
$$

and thus $k\left(u^{o}\right) \alpha \in F_{0}^{2}(\mathfrak{g}) W(L)^{(l)}$ for $\alpha \in V(L, \mathfrak{g})^{*}$. For $\widetilde{\beta} \in S^{1} L^{*}$ we have by (3.14)

$$
k\left(u^{\sigma}\right) \widetilde{\beta}=d_{W \otimes l+1}\left(u^{\sigma}(\beta)\right)-\sum_{j=1}^{l}\left(\beta^{(j)}-\beta^{(0)}\right) \otimes d t_{j}+\frac{1}{2} \beta\left[u^{\sigma}, u^{\sigma}\right] .
$$

Expansion along $u^{\sigma}=\sum_{j=0}^{l} t_{j} u_{j}$ gives by straightforward calculation

$$
\begin{aligned}
& k\left(u^{\sigma}\right) \widetilde{\beta}=\sum_{j=0}^{l} t_{j} \beta^{(j)}-\sum_{j=1}^{l}\left(\beta^{(j)}-\beta^{(0)}\right) \otimes d t_{j} \\
& \quad-\frac{1}{2} \sum_{j=1}^{l} t_{j} \beta\left[u_{j}-u_{0}, u_{j}-u_{0}\right]+\frac{1}{2} \sum_{i, j=1}^{l} t_{i} t_{j} \beta\left[u_{i}-u_{0}, u_{j}-u_{0}\right]
\end{aligned}
$$


For dual bases $x_{k}, x_{k}^{*}$ in $L$ and $L^{*}$ we obtain by the expansion formula

$$
-\beta\left[u_{i}, u_{j}\right]=\sum_{k} x_{k}^{*(i)} \wedge\left(\theta\left(x_{k}\right) \beta\right)^{(j)}
$$

the explicit expression

$$
\begin{aligned}
k\left(u^{o}\right) \widetilde{\beta} & =\sum_{j=0}^{l} t_{j} \widetilde{\beta}^{(j)}-\sum_{j=1}^{l}\left(\beta^{(j)}-\beta^{(0)}\right) \otimes d t_{j} \\
& +\frac{1}{2} \sum_{j=1}^{l} t_{j}\left\{\sum_{k}\left(x_{k}^{*(j)}-x_{k}^{*(0)}\right) \wedge\left(\left(\theta\left(x_{k}\right) \beta\right)^{(j)}-\left(\theta\left(x_{k}\right) \beta\right)^{(0)}\right)\right\} \\
& -\frac{1}{2} \sum_{i, j=1}^{l} t_{i} t_{j}\left\{\sum_{k}\left(x_{k}^{*(j)}-x_{k}^{*(0)}\right) \wedge\left(\left(\theta\left(x_{k}\right) \beta\right)^{(j)}-\left(\theta\left(x_{k}\right) \beta\right)^{(0)}\right)\right\} .
\end{aligned}
$$

This formula shows that all coefficients of $k\left(u^{\sigma}\right) \widetilde{\beta}$ are indeed elements of $F_{0}^{2}(\mathrm{~g})$ and the proof of Proposition 4.41 is complete.

REMARK 4.50. From the universal formula (4.48) we obtain for any family $\omega=\left(\omega_{0}, \cdots, \omega_{l}\right)$ of connections $\omega_{j}: W(\mathfrak{g}) \rightarrow E$ the following formula for $k\left(\omega^{\sigma}\right) \widetilde{\beta}, \widetilde{\beta} \in S^{1} \mathrm{~g}^{*}$

$$
\begin{aligned}
& k\left(\omega^{\sigma}\right) \widetilde{\beta}=\sum_{j=0}^{l} t_{j} k\left(\omega_{j}\right) \widetilde{\beta}-\sum_{j=1}^{l}\left(\omega_{j}-\omega_{0}\right) \beta \otimes d t_{j} \\
& -\frac{1}{2} \sum_{j=1}^{l} t_{j} \beta\left[\omega_{j}-\omega_{0}, \omega_{j}-\omega_{0}\right]+\frac{1}{2} \sum_{i, j=1}^{l} t_{i} t_{j} \beta\left[\omega_{i}-\omega_{0}, \omega_{j}-\omega_{0}\right] .
\end{aligned}
$$

In particular if $\widetilde{\beta} \in S^{1} \mathrm{~g}^{*}$ is invariant, i.e., $\widetilde{\beta} \in I(\mathfrak{g})^{2}$, then

$$
k\left(\omega^{\sigma}\right) \widetilde{\beta}=\sum_{j=0}^{l} t_{j} k\left(\omega_{j}\right) \widetilde{\beta}-\sum_{j=1}^{l}\left(\omega_{j}-\omega_{0}\right) \beta \otimes d t_{j} .
$$

Tне Номотору $\lambda^{1}(\theta)$. 4.53. The following application of the universal homotopy $\lambda^{1}$ is important.

Proposition 4.54. Let $\theta: L \rightarrow \mathfrak{g}$ be a linear retraction of $i: \mathfrak{g} \rightarrow L$.

(i) There exists a well-defined map

$$
\lambda^{1}(\theta): W(L)^{\cdot} \rightarrow W(L)^{-1}
$$

with the properties:

$$
\begin{aligned}
& d \lambda^{1}(\theta)+\lambda^{1}(\theta) d=k(\theta) \circ W(i)-\mathrm{id} \\
& \lambda^{1}(\theta): F_{0}^{2 p}(\mathfrak{g}) W(L) \rightarrow F_{0}^{2 p}(\mathfrak{g}) W(L) .
\end{aligned}
$$

(ii) If $\theta$ is h-equivariant for any subalgebra $\mathfrak{h} \subset \mathrm{g}$, then $\lambda^{1}(\theta)$ is a $V(\mathfrak{G})$-module homomorphism.

Proof. With the universal homotopy

$$
\lambda^{1}: W(L)^{\cdot} \rightarrow W\left(L^{2}\right)^{-1} \cong(W(L) \otimes W(L))^{-1}
$$


the definition of $\lambda^{1}(\theta)$ is

$$
\lambda^{1}(\theta)=(\mathrm{id}, k(\theta) \circ W(i)) \circ \lambda^{1} .
$$

To verify the stated properties, we identify the map

$$
(\mathrm{id}, k(\theta) \circ W(i)): W(L) \otimes W(L) \rightarrow W(L)
$$

as the functorial map induced in the sense of (4.22) by a linear map $\varphi: L \rightarrow L \times L$. For this purpose consider the composition $\varphi$ defined by the commutative diagram

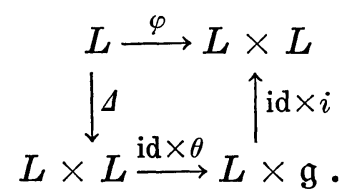

$\phi$ is a linear map over $\mathfrak{g}$ (with $\Delta \circ i: \mathfrak{g} \rightarrow L \times L$ the inclusion of $\mathfrak{g}$ in $L \times L)$. By functoriality we have for $k(\varphi): W(L \times L) \rightarrow W(L)$

$$
k(\varphi)=W(\Delta) \circ k(\mathrm{id} \times \theta) \circ k(\mathrm{id} \times i) .
$$

On $W(L) \otimes W(L) \cong W(L \times L)$ the map $W(\Delta)$ is the multiplication and hence for $a \otimes b \in W(L) \otimes W(L)$

$$
k(\varphi)(a \otimes b)=a_{.} k(\theta) W(i) b .
$$

It follows that

$$
k(\varphi)=(\mathrm{id}, k(\theta) \circ W(i)) .
$$

By Corollary 4.32 we have then

$$
k(\varphi): F_{0}^{2 p}(\mathfrak{g}) W(L \times L) \rightarrow F_{0}^{2 p}(\mathfrak{g}) W(L) .
$$

Together with Proposition 4.41 this implies the filtration property (4.56). The homotopy formula (4.55) follows from composition of the homotopy formula for $\lambda^{1}$ in (3.26), part (i) with the DG-homomorphism $k(\varphi)$. The proof of part (ii) follows from the fact that $\lambda^{1}$ is a map of $V(\mathfrak{g})$-modules, and $k(\theta)$ and hence also $k(\varphi)$ are maps of $V(\mathfrak{h})$-modules for $\mathfrak{h}$-equivariant $\theta$.

We finally consider the g-truncated relative Weil algebra

$$
\left\{\begin{array}{l}
W_{0}(L, \mathfrak{h})_{q}=W(L, \mathfrak{h}) / F_{0}^{2(q+1)}(\mathfrak{g}) W(L, \mathfrak{h}), \quad 0 \leqq q \\
W_{0}(L, \mathfrak{h})_{\infty}=W_{0}(L, \mathfrak{h})=W(L, \mathfrak{h}), \quad q=\infty .
\end{array}\right.
$$

For $L=g$ this coincides with the truncated relative Weil algebra with respect to the canonical filtration $F(\mathfrak{g})$ 


$$
\left\{\begin{array}{l}
W(\mathfrak{g}, \mathfrak{h})_{q}=W(\mathfrak{g}, \mathfrak{h}) / F^{2(q+1)}(\mathfrak{g}) W(\mathfrak{g}, \mathfrak{h}), \quad 0 \leqq q \\
W(\mathfrak{g}, \mathfrak{h})_{\infty}=W(\mathfrak{g}, \mathfrak{h}), \quad q=\infty
\end{array}\right.
$$

as considered in [14] [16]. We show that $W_{0}(L, \mathfrak{h})_{q}$ and $W(\mathfrak{g}, \mathfrak{h})_{q}$ are homotopy equivalent for all $0 \leqq q \leqq \infty$, and this functorially for linear maps $L^{\prime} \rightarrow L$ over $\mathfrak{g}$ which are $\mathfrak{h}$-equivariant. More precisely we have the following result.

TheORem 4.63. Let $\varphi: L^{\prime} \rightarrow L$ be a linear map such that

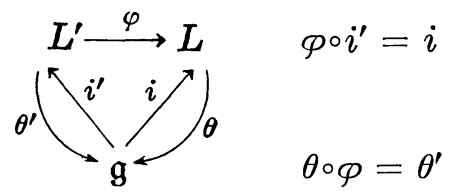

for the inclusions $i: \mathfrak{g} \rightarrow L, i^{\prime}: \mathfrak{g} \rightarrow L^{\prime}$ and retractions $\theta: L \rightarrow \mathfrak{g}, \theta^{\prime}: L^{\prime} \rightarrow$ g. Let $\mathfrak{h} \subset \mathfrak{g}$ and assume $\theta, \theta^{\prime}$ as well as $\varphi$ to be $\mathfrak{h}$-equivariant. Then for every $q, 0 \leqq q \leqq \infty$, there is a diagram

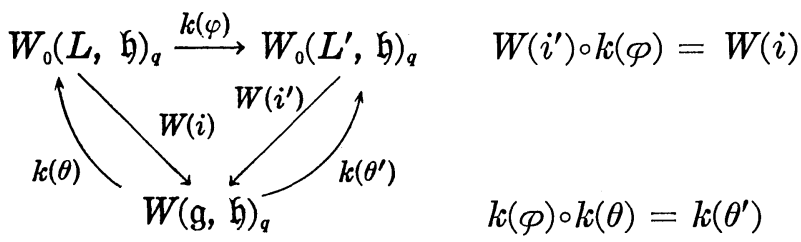

of DG-homomorphisms between the relative truncated Weil algebras with respect to the filtration $F_{0}(\mathrm{~g})$. The maps $k(\theta), W(i)$ resp. $k\left(\theta^{\prime}\right)$, $W\left(i^{\prime}\right)$ are inverse homotopy equivalences. Hence $k(\varphi)$ induces an isomorphism in cohomology which is independent of the linear map $\varphi: L^{\prime} \rightarrow L$ over g.

Proof. By functoriality diagram (4.64) induces a diagram

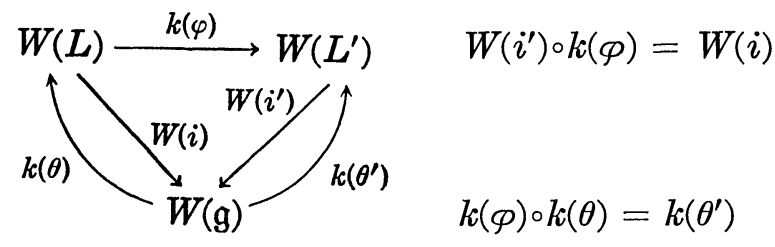

of $\mathfrak{h}$-DG-homomorphisms. They preserve the filtrations $F_{0}(\mathfrak{g})$ by (4.32). Clearly $W(i) \circ k(\theta)=\mathrm{id}$, and $k(\theta) \circ W(i) \simeq \mathrm{id}$ according to (4.55). The homotopy $\lambda^{1}(\theta)$ preserves by (4.56) the filtration $F_{0}(\mathfrak{g})$ on $W(L)$. Similarly for $L^{\prime}$. The desired result follows by passing everywhere to the $h$-basic subalgebras and to the quotients by the filtration ideals $F_{0}^{2(q+1)}(\mathfrak{g})$.

For a convenient reformulation consider a pair $(\mathfrak{g}, \mathfrak{h})$ of Lie algebras 
admitting an $\mathfrak{h}$-equivariant retraction $\mathfrak{g} \rightarrow \mathfrak{h}$. Denote $\mathscr{L}_{(g, \mathfrak{b})}$ the category of Lie algebras $L$ over $\mathfrak{g}$ admitting an $\mathfrak{h}$-equivariant retraction $L \rightarrow \mathfrak{g}$. Then we have the following result.

CoROLLARY 4.67. (i) The filtered functor $W_{0}(-, \mathfrak{h})_{q}$ on the category $\mathscr{L}_{(8,6)}$ is homotopy equivalent to the constant functor given by the canonically filtered $\mathrm{g}$-DG-algebra $W(\mathfrak{g}, \mathfrak{h})_{q}$.

(ii) The spectral functor associated to the filtration $F_{0}^{2 p}(\mathfrak{g})$ on $W_{0}(-, \mathfrak{h})_{a}$ is naturally equivalent to the constant functor given by the spectral sequence associated to the canonical filtration $F(\mathfrak{g})$ on $W(\mathfrak{g}, \mathfrak{h})_{q}$.

Proof. (i) was proved in Theorem 4.63. Part (ii) follows by the same argument, since $W(i), k(\theta)$, and $\lambda^{1}(\theta)$ induce homotopy equivalences of the associated graded algebras

$$
G_{0} W_{0}(L, \mathfrak{g})_{q} \simeq G W(\mathfrak{g}, \mathfrak{h})_{q},
$$

and hence isomorphisms of the $E_{r}$-terms for $r \geqq 1$.

REMARK 4.69. No such results hold with respect to the canonical filtration $F(\mathfrak{g})$ on $W(L, \mathfrak{h})_{q}$. The maps

$$
W(L) \underset{k(\theta)}{\stackrel{W(i)}{\rightleftarrows}} W(\mathrm{~g})
$$

are still homotopy-equivalences. But the homotopy $k(\theta) \circ W(i) \simeq \mathrm{id}$ is not compatible with the canonical filtration, and therefore does not pass to the quotients by the filtration ideals with respect to $F(\mathrm{~g})$. In fact there is not even an isomorphism of the homologies $H\left(W(L, \mathfrak{h}) / F^{2(q+1)}(\mathfrak{g}) W(L, \mathfrak{h})\right)$ and $H\left(W(\mathfrak{g}, \mathfrak{h}) / F^{2(q+1)}(\mathfrak{g}) W(\mathfrak{g}, \mathfrak{h})\right)$. To see this let e.g., $\mathfrak{h}=\mathfrak{g}$. Then

$$
W(L, \mathfrak{g}) \simeq W(\mathfrak{g}, \mathfrak{g})=I(\mathfrak{g}) \text {. }
$$

But

$$
H\left(W(L, \mathfrak{g}) / F^{2(q+1)}(\mathfrak{g}) W(L, \mathfrak{g})\right) \text { and } I(\mathfrak{g}) / F^{2(q+1)}(\mathfrak{g}) I(\mathfrak{g})
$$

are certainly not isomorphic. E.g., for $\mathfrak{g}=0$ the second algebra equals the ground field, whereas the first algebra is highly non-trivial [3] [6] [10] [12].

SigNificANCE of $W_{0}(-, \mathfrak{h})_{q} 4.70$. In the next section we will establish the appropriate generalizations of the statements 4.63 and 4.67 for the functors $W_{s}(-, \mathfrak{h})_{q}$. But first we wish to give a geometric interpretation for the complex $W_{0}(-, \mathfrak{h})_{q}$. For this purpose we return to the geometric context explained in the introduction. More specifically we consider a foliated $\bar{G}$-bundle $\bar{P} \stackrel{\vec{\pi}}{\rightarrow} X$. The foliation $L_{\bar{G}}$ on $X$ is the 
quotient of a foliation $L$ on $\bar{P}$ which is $\bar{G}$-invariant i.e., $L_{\bar{G}}=L / \bar{G}$. For a closed subgroup $G \subset \bar{G}$ there is then defined on $M=\bar{P} / G$ a quotient foliation $L_{G}=L / G$. The projection $\bar{P}=P \rightarrow \bar{P} / G$ is itself a foliated $G$ bundle $P \stackrel{\pi}{\rightarrow} M$. Thus there is a factorization

$$
\begin{gathered}
\bar{P}=\underset{\pi \searrow}{P \stackrel{\bar{\pi}}{\longrightarrow}} X=\bar{P} / \bar{G} \\
M=\overline{\bar{T}} / G
\end{gathered}
$$

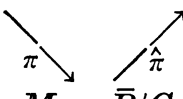

of the foliated bundle map $\bar{\pi}$ into the foliated bundle map $\pi$ and the $\bar{G} / G$-fibration $\hat{\pi}$. This situation has been extensively discussed in [18], Section 3, to which we refer for more details on the following discussion. Let $l$ be the codimension of the foliation $L$ on $X, q=\operatorname{dim} \overline{\mathrm{g}} / \mathrm{g}$. Then the foliation $L_{G}$ on $M$ has codimension $q+l$. Let $H \subset G$ be a closed subgroup and $\theta$ an $H$-equivariant splitting of the exact sequence

$$
0 \longrightarrow \mathrm{g} \stackrel{\theta}{\longrightarrow} \overline{\mathrm{g}} \longrightarrow \overline{\mathrm{g}} / \mathrm{g} \longrightarrow 0 .
$$

An adapted connection $\bar{\omega}$ in the $\bar{G}$-bundle $\bar{P}$ leads to an adapted connection $\omega=\theta \circ \bar{\omega}$ in the G-bundle $P$. This in turn leads to the following commutative diagram

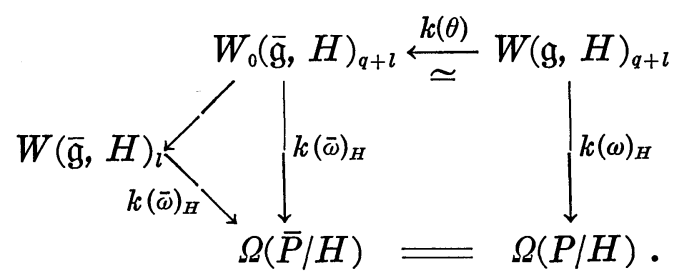

The vertical map on the RHS is the Weil homomorphism of $\omega$ on $P$. The map $k(\overline{\boldsymbol{\omega}})_{H}: W(\overline{\mathfrak{g}}, H)_{l} \rightarrow \Omega(\bar{P} / H)$ is the Weil homomorphism of $\overline{\boldsymbol{\omega}}$ on $\bar{P}$, annihilating the filtration ideal $F^{2(l+1)}(\overline{\mathfrak{g}}) W(\overline{\mathfrak{g}}, H)$. The point of diagram 4.72 is that this same map on $W(\overline{\mathrm{g}}, H)$ also annililates the filtration ideal $F_{0}^{2(q+l+1)}(\mathfrak{g}) W(\overline{\mathfrak{g}}, H)$, since

$$
F_{0}^{2(q+p)}(\mathfrak{g}) W(\overline{\mathfrak{g}}, H) \subset F^{2 p}(\overline{\mathfrak{g}}) W(\overline{\mathfrak{g}}, H), \quad p \geqq 0 .
$$

The effect of this is that the Weil homomorphism $k(\omega)$ of $\omega$ can be realized directly by $k(\bar{\omega})$ on the complex $W_{0}(\overline{\mathfrak{g}}, H)_{q+l}$. This is the geometric significance of the filtration $F_{0}(\mathfrak{g})$ on $W(\overline{\mathfrak{g}}, H)$.

We turn next to a generalization of these results to the functors $W_{s}(-, \mathfrak{h})_{q}, s>0$. 
5. Isomorphism Theorems. In this section we prove the main result of this paper, namely the homology equivalence of all truncated relative ss Weil algebras $W_{s}(L, \mathfrak{h})_{q}$ for $s \geqq 0$.

First we generalize the Definition (4.29) of the filtration $F_{0}(g)$ on the functor $W_{0}=W$ as follows. Let $s>0$ and $W_{s}(L)$ the $g$-DG-algebra defined in (2.22). Then

$$
F_{s}^{2 p}(\mathfrak{g}) W_{s}(L)=\underset{l \geqq 0}{\Perp} F_{s-1}^{2 p}(\mathfrak{g}) W_{s-1}\left(L^{l+1}\right)
$$

defines recursively an even filtration on $W_{s}(L)$.

LEMMA 5.2. $\quad F_{s}(\mathfrak{g}) W_{s}(L)$ satisfies the following properties:

(i ) $F_{s}(\mathrm{~g}) W_{s}(L)$ is functorial for Lie homomorphisms $L^{\prime} \rightarrow L$ over $\mathrm{g}$;

(ii) $F_{s}(\mathrm{~g}) W_{s}(L)$ is a decreasing, bihomogeneous and multiplicative filtration by $\mathfrak{g}$-DG-ideals;

(iii) $F_{s}(\mathfrak{g}) W_{s}$ is preserved under the face and degeneracy maps

$$
\varepsilon_{i}^{l}: W_{s}\left(L^{l+1}\right) \rightarrow W_{s}\left(L^{l+2}\right), \quad \mu_{j}^{l}: W_{s}\left(L^{l+2}\right) \rightarrow W_{s}\left(L^{l+1}\right) .
$$

Proof. (i) For $s=0$ this property holds by part (ii) of 4.32 (even for linear maps $L^{\prime} \rightarrow L$ over g). From (5.1) it is then clear that the functoriality holds for all $s \geqq 0$. (ii), (iii) are proved by induction on $s$ (the case $s=0$ is clear). As the operators $i(x), \theta(x)$ for $x \in \mathfrak{g}$ leave $W_{s}(L)^{l}=W_{s-1}\left(L^{l+1}\right)$ invariant, so are the subspaces $F_{s}^{2 p} W_{s}(L)$ by (5.1). We can assume that $d_{W_{s-1}}$ leaves $F_{s-1}^{2 p} W_{s-1}\left(L^{l+1}\right)$ invariant and that the face and degeneracy maps satisfy

$$
\begin{aligned}
& \varepsilon_{i}^{l}: F_{s-1}^{2 p} W_{s-1}\left(L^{l+1}\right) \rightarrow F_{s-1}^{2 p} W_{s-1}\left(L^{l+2}\right) \\
& \mu_{j}^{l}: F_{s-1}^{2 p} W_{s-1}\left(L^{l+2}\right) \rightarrow F_{s-1}^{2 p} W_{s-1}\left(L^{l+1}\right) .
\end{aligned}
$$

It follows that $\delta=\sum_{i=0}^{l+1}(-1)^{i} \varepsilon_{i}^{l}: F_{s}^{2 p} W_{s}(L) \rightarrow F_{s}^{2 p} W_{s}(L)$ and therefore also

$$
d_{W_{s}(L)}=\delta+(-1)^{l} d_{W_{s-1}\left(L^{l+1}\right)}: F_{s}^{2 p} W_{s}(L) \rightarrow F_{s}^{2 p} W_{s}(L) .
$$

The face and degeneracy maps obviously preserve $F_{s} W_{s}$. The multiplicativity follows from the commutative diagram below (for the notations see the definition of the multiplication in the proof of 2.14).

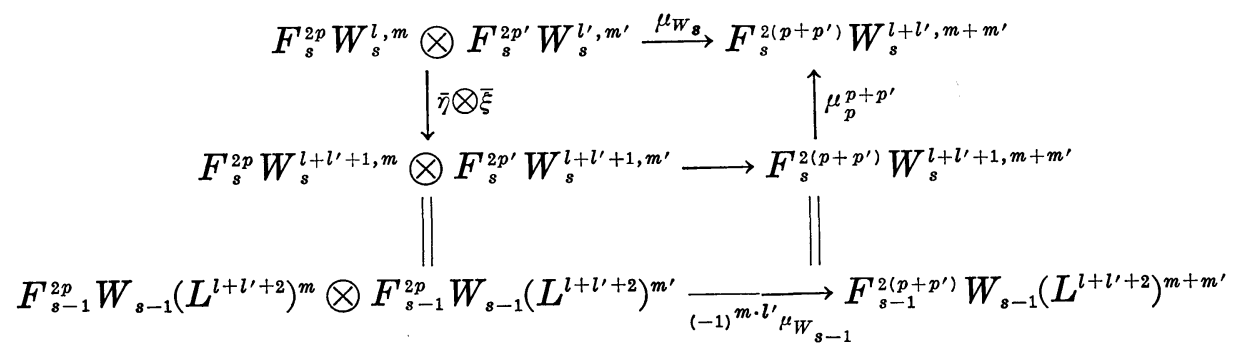


Since the vertical maps (defined by face maps) are filtration preserving, and $\mu_{W_{s-1}}$ does so by induction assumption, so does $\mu_{W_{s}}$. This completes the proof of 5.2 .

Thus we can consider more generally than (4.61) the $g$-truncated relative ss Weil algebras

$$
\left\{\begin{array}{l}
W_{s}(L, \mathfrak{h})_{q}=W_{s}(L, \mathfrak{h}) / F_{s}^{2(q+1)}(\mathfrak{g}) W_{s}(L, \mathfrak{h}), \quad 0 \leqq q \\
W_{s}(L, \mathfrak{h})_{\infty}=W_{s}(L, \mathfrak{h}), \quad q=\infty
\end{array}\right.
$$

For $s>0$ the natural projection (2.24) induces DG-maps

$$
\rho_{s}(L, \mathfrak{h}): W_{s}(L, \mathfrak{h})_{q} \rightarrow W_{s-1}(L, \mathfrak{h})_{q}, \quad 0 \leqq q \leqq \infty .
$$

The following theorem was originally stated in [13] [15] for $L=\mathfrak{g}$ under the assumption that $(\mathfrak{g}, \mathfrak{h})$ is a reductive pair of Lie algebras. The assumption we need is only the existence of an $\mathfrak{h}$-equivariant retraction $\mathfrak{g} \rightarrow \mathfrak{h}$.

THEOREM 5.5. Let $L$ be a Lie algebra over $\mathfrak{g}$ admitting an h-equivariant retraction $L \rightarrow \mathrm{g}$. Then for every $q, 0 \leqq q \leqq \infty$, and every $s>0$ the commutative diagram of DG-homomorphisms

$$
\begin{gathered}
W_{s}(L, \mathfrak{h})_{q} \stackrel{\rho_{s}(L, \mathfrak{h})}{\longrightarrow} W_{s-1}(L, \mathfrak{h})_{q} \\
\qquad W_{s}(i, \mathfrak{h}) \\
W_{s}(\mathfrak{g}, \mathfrak{h})_{q} \stackrel{\rho_{s}(\mathfrak{g}, \mathfrak{h})}{\longrightarrow} W_{s-1}(\mathfrak{g}, \mathfrak{h})_{q}
\end{gathered}
$$

induces a commutative diagram of homology-isomorphisms. This diagram is natural for Lie homomorphisms $L^{\prime} \rightarrow L$ of Lie algebras over $\mathrm{g}$.

Together with Theorem 4.63 this establishes the following fact.

Corollary 5.7 [13]. Let $0 \leqq q \leqq \infty$. Then the functors $H\left(W_{s}(L, \mathfrak{h})_{q}\right)$, $s \geqq 0$ on the category $\mathscr{L}_{(8,6)}$ of Lie algebras over $\mathrm{g}$ are all naturally equivalent to the constant functor given by the algebra $H\left(W(\mathfrak{g}, \mathfrak{h})_{q}\right)$.

For $q=\infty$ this leads to isomorphisms

$$
H\left(W_{s}(L, \mathfrak{h})\right) \cong H(W(\mathfrak{g}, \mathfrak{h})) \cong I(\mathfrak{h}), \quad s \geqq 0 .
$$

The last isomorphism is induced by the restriction $W(\mathfrak{g}, \mathfrak{h}) \rightarrow W(\mathfrak{h}, \mathfrak{h})=$ $I(\mathfrak{G})$, which is a homotopy equivalence by formula (4.55) for the inclusion $\mathfrak{h} \subset \mathfrak{g}$ and an $h$-equivariant retraction $\mathfrak{g} \rightarrow \mathfrak{h}$. For $\mathfrak{h}=0$ in particular this shows that

$$
\left.H\left(W_{s}(L)\right) \cong H(W(\mathrm{~g})) \cong k \quad \text { (ground field }\right)
$$


i.e., the cohomological triviality of the ss Weil algebra and the ordinary Weil algebras.

For $0 \leqq q \leqq \infty$ and $\mathfrak{h}=\mathfrak{g}$ we have further by (5.7) the isomorphisms

$$
H\left(W_{s}(L, \mathfrak{g})_{q}\right) \cong H\left(W(\mathfrak{g}, \mathfrak{g})_{q}\right) \cong I(\mathfrak{g})_{q}, \quad s \geqq 0 .
$$

Proof OF THEOREM 5.5. We consider the following properties for fixed $q, 0 \leqq q \leqq \infty$.

$Q_{8}$ : for every $0 \leqq t \leqq s$ the natural restriction homomorphism

$$
W_{t}(i, \mathfrak{h}): W_{t}(L, \mathfrak{h})_{q} \rightarrow W_{t}(\mathfrak{g}, \mathfrak{h})_{q}
$$

induces a homology isomorphism.

$P_{s}:$ for every $1 \leqq t \leqq s$ the natural projection

$$
\rho_{t}(L, \mathfrak{h}): W_{t}(L, \mathfrak{h})_{q} \rightarrow W_{t-1}(L, \mathfrak{h})_{q}
$$

induces a homology isomorphism.

The statement $Q_{0}$ was proved in Theorem 4.63. We shall prove the following implications:

(i) $Q_{s} \Rightarrow P_{s+1}$,

(ii) $Q_{0}$ and $P_{s+1} \Rightarrow Q_{s+1}$.

This will then complete the proof of Theorem 5.5.

To prove (i) we consider the spectral sequence associated to the following filtration on $W_{s+1}(L, \mathfrak{h})_{q}$ :

$$
{ }^{\prime} F^{l} W_{s+1}(L, \mathfrak{h})_{q}=\underset{m \geq l}{\Perp} W_{s}\left(L^{m+1}, \mathfrak{h}\right)_{q} .
$$

This is a decreasing multiplicative filtration with graded algebra

$$
{ }^{\prime} E_{0}^{l, \cdot}={ }^{\prime} G^{l} W_{s+1}^{\cdot}(L, \mathfrak{h})_{q}=W_{s}^{:}\left(L^{l+1}, \mathfrak{h}\right)_{q}
$$

and $d_{0}=G\left(d_{W_{s+1}}\right)=(-1)^{l} d_{W_{s}\left(L^{l+1}\right)}$. It follows that

$$
{ }^{\prime} E_{1}^{l, \cdot}=H\left(W_{s}\left(L^{l+1}, \mathfrak{h}\right)_{q}\right) \text {. }
$$

We can consider the assignment $\sigma_{l} \mapsto H\left(W_{s}\left(L^{l+1}, \mathfrak{h}\right)_{q}\right)$ for $l \geqq 0$ as the local system of coefficients $\boldsymbol{H}\left(W_{s}(\Delta L, \mathfrak{h})_{q}\right)$ on the ss Pt. Then

$$
{ }^{\prime} E_{1}^{l,},=C^{l}\left(\mathrm{Pt}, \boldsymbol{H}\left(W_{s}(\Delta L, \mathfrak{h})_{q}\right)\right) .
$$

The differential $d_{1}:{ }^{\prime} E_{1}^{l,} \rightarrow{ }^{\prime} E_{1}^{l+1, .}$ of bidegree $(1,0)$ corresponds to $\bar{\delta}_{l}=$ $\sum_{j=0}^{l+1}(-1)^{j} \bar{\varepsilon}_{j}^{l}$. We claim that as a consequence of $Q_{s}$ the local system $\boldsymbol{H}\left(W_{s}(\Delta L, \mathfrak{h})_{q}\right)$ is isomorphic to the constant local system defined by 
$H\left(W(\mathfrak{g}, \mathfrak{h})_{q}\right)$. For this purpose consider for $l \geqq 0$ the commutative diagram of Lie homomorphisms

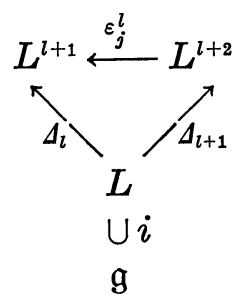

where $\Delta_{l}, \Delta_{l+1}$ denote the respective diagonal maps and $j=0, \cdots, l+1$. By functoriality there is then a commutative diagram

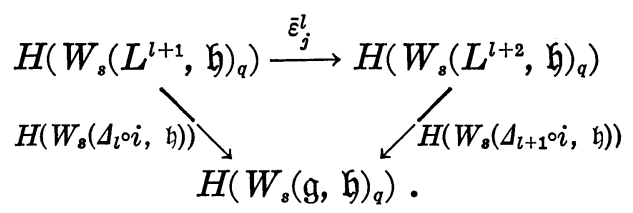

By the assumption $Q_{g}$, the maps into $H\left(W_{s}(\mathfrak{g}, \mathfrak{h})_{q}\right)$ are isomorphisms. It follows that the map $\bar{\varepsilon}_{j}^{l}$ is an isomorphism which does not depend on $j=0, \cdots, l+1$. This proves the constancy of the system $\boldsymbol{H}\left(W_{s}(\Delta L, \mathfrak{h})_{q}\right)$. In particular the face maps $\bar{\varepsilon}_{j}^{l}$ correspond to the identity under the identifications

$$
H\left(W_{8}\left(L^{l+1}, \mathfrak{h}\right)\right) \cong H\left(W_{8}(\mathfrak{g}, \mathfrak{h})_{q}\right) .
$$

It follows that

$$
\bar{\delta}_{l}=\sum_{j=0}^{l+1}(-1)^{j} \mathrm{id}=\left\{\begin{array}{llll}
\text { id } & \text { for } & l & \text { odd } \\
0 & \text { for } & l & \text { even }
\end{array}\right\} .
$$

Therefore

$$
{ }^{\prime} E_{2}^{l, \cdot}=H_{\delta}^{l}\left(\mathrm{Pt}, \boldsymbol{H}^{\cdot}\left(W_{s}(\Delta L, \mathfrak{h})\right)_{q}\right) \cong\left\{\begin{array}{cc}
0 & \text { for } l>0 \\
H\left(W_{s}(\mathfrak{g}, \mathfrak{h})_{q}\right) & \text { for } l=0
\end{array}\right.
$$

and the spectral sequence 'of the filtered algebra (5.11) collapses. The edge map

$$
H\left(W_{s+1}(L, \mathfrak{h})_{q}\right) \rightarrow H\left(W_{s}(L, \mathfrak{h})_{q}\right)
$$

is therefore an isomorphism. But this map is induced by the natural projection $\rho_{s+1}(L, \mathfrak{h})$. This establishes the implication $Q_{s} \Rightarrow P_{s+1}$.

To prove the implication (ii), consider the commutative diagram of DG-homomorphisms 


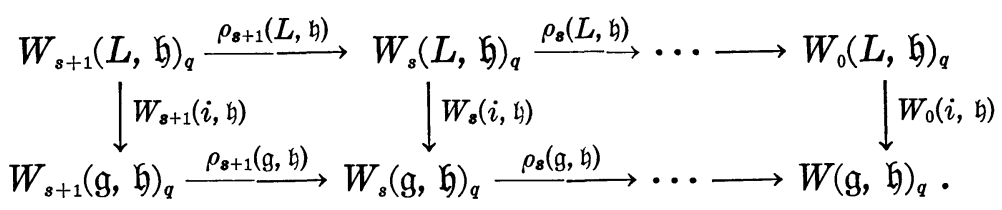

Clearly property $Q_{0}$ together with $P_{s+1}$ implies then that all vertical maps induce homology isomorphisms, i.e., property $Q_{s+1}$. This finishes the proof of Theorem 5.5.

REMARK 5.19. The argument in the proof just completed establishes more generally the following fact. Let $F: \mathscr{L}_{g} \leadsto \mathscr{A}$ be a contravariant functor with values in the category $\mathscr{A}$ of DG-algebras. Define recursively a sequence of functors $F_{s}, s \geqq 0\left(F_{0}=F\right)$ as explained at the end of Section 2. If we apply this construction to the functor $F_{0}=W_{0}(-, \mathfrak{h})_{q}$, it is easy to verify that $F_{s}=W_{s}(-, \mathfrak{h})_{q}$. If $F_{0}$ satisfies property $Q_{0}$, i.e., if the homology functor $H(F)$ on $\mathscr{L}_{\mathrm{g}}$ is naturally equivalent to the constant functor defined by the algebra $H(F(\mathrm{~g})$ ) (the value of $H(F)$ on the initial object of $\mathscr{L}_{g}$ ), then more generally all functors $F_{s}, s \geqq 0$ satisfy the same property. In other words the implication $Q_{0} \Rightarrow Q_{s}$, $s \geqq 0$ follows by a general spectral sequence argument, whereas the property $Q_{0}$ for $F_{0}$ may or may not hold. For the case of the functor $F_{0}=W_{0}(-, \mathfrak{h})_{q}$ on the category $\mathscr{L}_{(8, \mathfrak{h})}$ the property $Q_{0}$ was established in Section 4 (Theorem 4.63 and Corollary 4.67), whereas the arguments in this section are of the general nature just explained.

We turn now to the map $\lambda: W \rightarrow W_{1}$. With the Definition (5.1) of $F_{1}^{2 p}(\mathfrak{g}) W_{1}(L)$ Proposition 4.41 clearly translates to the fact

$$
\lambda: F_{0}^{2 p}(\mathfrak{g}) W(L) \rightarrow F_{1}^{2 p}(\mathfrak{g}) W_{1}(L), \quad p \geqq 0 .
$$

It follows that $\lambda$ induces an additive DG-map

$$
\lambda: W_{0}(L, \mathfrak{h})_{q} \rightarrow W_{1}(L, \mathfrak{h})_{q}, \quad 0 \leqq q \leqq \infty .
$$

THEOREM 5.21. The induced homology map

$$
\lambda_{*}: H\left(W_{0}(L, \mathfrak{h})_{q}\right) \rightarrow H\left(W_{1}(L, \mathfrak{h})_{q}\right)
$$

is a multiplicative isomorphism inverting the homology isomorphism $\left(\rho_{1}\right)_{*}$ induced by the canonical projection $\rho_{1}(L, \mathfrak{h}): W_{1}(L, \mathfrak{h})_{q} \rightarrow W_{0}(L, \mathfrak{h})_{q}$.

Proof. By part (ii) of Theorem 3.26 we have $\rho_{1} \circ \lambda=$ id: $W_{0}(L) \rightarrow$ $W_{0}(L)$. Since both $\lambda$ and $\rho_{1}$ are filtration preserving, this also holds on $W_{0}(L, \mathfrak{h})_{q}$. But $\left(\rho_{1}\right)_{*}$ is already known to be an isomorphism by Theorem 5.5. Therefore the same holds for the one-sided inverse $\lambda_{*}$.

REMARK. While by the above argument the homology map $\lambda_{*}$ is 
an isomorphism of algebras, it is worth emphasizing that $\lambda$ itself is definitely not multiplicative (except for $\mathfrak{g}=\boldsymbol{R}$ ). For this it suffices to observe that for linearly independent $\alpha, \beta \in \mathrm{g}^{*}$ in fact $\lambda(\widetilde{\alpha}) \lambda(\widetilde{\beta}) \neq \lambda(\widetilde{\beta}) \lambda(\widetilde{\alpha})$ in $W_{1}(\mathfrak{g})$. Since $\lambda$ is defined on the commutative algebra $W(\mathfrak{g})$, it cannot be multiplicative.

REMARK 5.22. The isomorphism $\lambda_{*}$ of Theorem 5.21 as well as the isomorphisms of Theorem 5.5 are applied in the geometric context to pairs $(G, H)$ of Lie groups, $H \subset G$ a closed subgroup. The passage to h-basic elements is then replaced by the passage to $H$-basic elements, which leads to algebras $W_{s}(L, H)_{q}, W_{s}(g, H)_{q}$ etc. We always assume $G$ connected and $H$ with finitely many connected components. For the action of the component group $\Gamma=H / H_{0}$ we have then for the invariants

$$
W_{s}(\mathfrak{g}, \mathfrak{h})_{q}^{\Gamma}=W_{s}(\mathfrak{g}, H)_{q} .
$$

Since the cohomology of $\Gamma$ is trivial, it follows that

$$
H\left(W_{s}(\mathfrak{g}, H)_{q}\right)=H\left(W_{s}(\mathfrak{g}, \mathfrak{h})_{q}\right)^{r} .
$$

As the maps $\rho_{s}(\mathfrak{g}, \mathfrak{h})_{*}$ in Theorem 5.5 are isomorphisms which are $\Gamma$-equivariant, it follows that the corresponding maps

$$
\rho_{s}(\mathrm{~g}, H)_{*}: H\left(W_{s}(\mathrm{~g}, H)_{q}\right) \rightarrow H\left(W_{s-1}(\mathfrak{g}, H)_{q}\right)
$$

are also isomorphisms. That

$$
\lambda_{*}: H\left(W(\mathfrak{g}, H)_{q}\right) \rightarrow H\left(W_{1}(\mathfrak{g}, H)_{q}\right)
$$

is an isomorphism follows again from the fact that it inverts $\rho_{1}(\mathfrak{g}, H)_{*}$.

Corollary 4.32, (i) has the following generalization to local systems $\boldsymbol{E}$ of $\mathrm{g}$-DG-algebras on a ss set $S$. The canonical $\mathfrak{g}$-filtration on $C(S, \boldsymbol{E})$ is given by

$$
F^{p}(\mathfrak{g}) C(S, E)=C\left(S, F^{p}(\mathfrak{g}) \boldsymbol{E}\right) .
$$

Proposition 5.24. Let $\omega=\left(\omega_{j}\right)_{j \in S_{0}}$ be a family of linear maps $\omega: L^{*} \rightarrow E_{j}^{1}, \quad j \in S_{0}$ satisfying the conditions (4.8) (4.9) in Proposition 4.7. Then the Weil homomorphism (3.30) has the filtration property

$$
k_{1}(\boldsymbol{\omega}): F_{1}^{2 p}(\mathrm{~g}) W_{1}(L) \rightarrow F^{p}(\mathfrak{g}) C(S, E), \quad p \geqq 0 .
$$

Proof. Together with the Definition (5.1) of $F_{1}^{2 p}(\mathfrak{g}) W_{1}(L)$ and the definition of $k_{1}(\omega)$ as a coefficient map this follows from part (i) of Corollary 4.32.

CoROLLARY 5.25. Let the situation be as above. Then the difference 
map $\lambda_{E}(\omega)$ has the filtration property

$$
\lambda_{E}(\boldsymbol{\omega}): F_{0}^{2 p}(\mathfrak{g}) W(L) \rightarrow F^{p}(\mathrm{~g}) C(S, E), \quad p \geqq 0 .
$$

Proof. Since by (3.31) the map $\lambda_{E}(\omega)$ is the composition $k_{1}(\omega) \circ \lambda$ : $W(L) \rightarrow C(S, E)$, this filtration property follows from (5.20) and (5.24).

REMARK 5.26. The last facts imply the following. If $\boldsymbol{E}$ is a local system with the property that $F^{q+1}(\mathfrak{g}) \boldsymbol{E}=0$, there is a commutative diagram of DG-maps

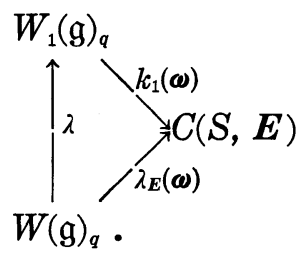

Since $\lambda$ induces a homology isomorphism, the maps $k_{1}(\omega)$ and $\lambda_{E}(\omega)$ are homologically equivalent. The same facts hold if $\boldsymbol{E}$ is equipped with any finite filtration such that $k_{1}(\omega)$ has the filtration property 5.24. This is in particular the case for a family $\omega$ of local adapted connections in a foliated bundle, as discussed in the remainder of this paper.

6. Generalized characteristic homomorphism. In this Section we apply the preceding results to the geometric context described in the introduction, where all these constructions originated (see [12] to [15]). For a foliated $G$-bundle $P \stackrel{\pi}{\rightarrow} M$, ( $G$ connected), an open covering $\mathscr{U}=$ $\left(U_{j}\right)_{j \in J}$ of $M$ such that $P / U_{j}$ admits an adapted connection $\omega_{j}$, the family $\omega=\left(\omega_{j}\right)$ on $P / \mathscr{U}$ defines then a generalized Weil homomorphism

$$
k_{1}(\omega): W_{1}^{*}(\mathrm{~g}) \rightarrow \check{C}^{\bullet}\left(\mathscr{C}, \pi_{*} \Omega_{P}^{*}\right) \text {. }
$$

We refer to $(3.30)$ for the construction of $k_{1}(\omega)$. The notations are those of the introduction. We write in particular $\breve{C}\left(\mathscr{C}, \pi_{*} \Omega_{P}^{*}\right)=C\left(N(\mathscr{U}), \pi_{*} \Omega_{P}^{*}\right)$, where $N(\mathscr{C})$ is the ss set given by the nerve of the covering $\mathscr{U}$. The map $k_{1}(\omega)$ embodies the idea of constructing the characteristic classes of $P$ out of local connections $\omega_{j}$ on $P / U_{j}$ for $\mathscr{C}=\left(U_{j}\right)_{j \in J}$. The early papers of Koszul [21] [22] already propagate the idea that the characteristic classes of a principal bundle can be constructed out of its transition functions. This corresponds to the choice of a family of local connections which locally trivialize the bundle.

To explain the filtration phenomena for the map (6.1), we first observe that the target carries a filtration defined by the normal bundle $Q=T_{M} / L$ of the foliation $L \subset T_{M}$. More precisely let $\mathbb{Q}^{*}$ denote the 
sheaf of sections of the dual bundle $Q^{*}$. The powers of the differential ideal $\mathscr{Q}^{*} . \pi_{*} \Omega_{P}^{*}$ define a filtration

$$
F^{p} \breve{C}\left(\mathscr{U}, \pi_{*} \Omega_{P}^{*}\right)=\check{C}\left(\mathscr{U}, \wedge^{p} \mathscr{Q}^{*} . \pi_{*} \Omega_{P}^{*}\right) \text { for } p \geqq 0 \text {. }
$$

This is a decreasing filtration by $\mathfrak{g}$-DG-ideals on $\check{C}$. With $q$ the codimension of $L \subset T_{M}$ we have then

$$
F^{q+1} \check{C}\left(\mathscr{C}, \pi_{*} \Omega_{P}^{*}\right)=0 .
$$

Observe that the codimension $q$ has to be taken as the rank of $\mathbb{Q}^{*}$ over the underlying structure sheaf $\mathcal{O}$, and then $\wedge^{q+1} \mathscr{Q}^{*}=0$, which implies (6.3). Thus e.g., in the holomorphic context $q$ denotes the complex codimension of $L \subset T_{M}$. The crucial filtration property of $k_{1}(\omega)$ is then as follows.

Proposition 6.4.

$$
k_{1}(\omega) F_{1}^{2 p}(\mathfrak{g}) W_{1}^{*}(\mathfrak{g}) \subset F^{p} \check{C}\left(\mathscr{U}, \pi_{*} \Omega_{\dot{P}}\right) \quad \text { for } \quad p \geqq 0 .
$$

PRoof. By (3.29), (3.30) the map $k_{1}(\omega)$ is determined by the coefficient maps

$$
k\left(\omega_{o}\right): W\left(\mathrm{~g}^{l+1}\right) \rightarrow \Gamma\left(U_{o}, \pi_{*} \Omega_{P}^{*}\right),
$$

where $\sigma=\left(i_{0}, \cdots, i_{l}\right) \in N(\mathscr{U})_{l}, l \geqq 0$. The filtration $F_{1}^{2 p}(\mathfrak{g})$ is determined on $W\left(\mathrm{~g}^{l+1}\right)=W_{1}^{l}(\mathrm{~g})$ by $F_{0}^{2 p}(\mathrm{~g}) W\left(\mathrm{~g}^{l+1}\right)$ (see (5.1)). Using (4.43), (6.2) and the multiplicativity of $k\left(\omega_{\sigma}\right)$, it is sufficient to verify the following filtration properties:

$$
\begin{gathered}
k\left(\omega_{o}\right)\left(\alpha^{(j)}-\alpha^{(k)}\right) \in \Gamma\left(U_{\left(i_{j}, i_{k}\right)}, \mathbb{Q}^{*} \cdot \pi_{*} \Omega_{P}\right)=\Gamma\left(\pi^{-1}\left(U_{\left(i_{j}, i_{k}\right)}, \pi^{*} \mathbb{Q}^{*} \cdot \Omega_{P}^{*}\right)\right. \\
k\left(\omega_{o}\right) \widetilde{\beta}^{(j)} \in \Gamma\left(U_{i_{j}}, \mathbb{Q}^{*} \cdot \pi_{*} \Omega_{P}^{*}\right)=\Gamma\left(\pi^{-1}\left(U_{i_{j}}\right), \pi^{*} \mathbb{Q}^{*} \cdot \Omega_{P}^{*}\right)
\end{gathered}
$$

for $\alpha \in \wedge^{1}\left(\mathrm{~g}^{*}\right)$ and $\widetilde{\beta} \in S^{1}\left(\mathrm{~g}^{*}\right)$ (see (4.46) for the notations). Concerning (6.5) we have $k\left(\omega_{o}\right)\left(\alpha^{(j)}-\alpha^{(k)}\right)=\omega_{i_{j}}(\alpha)-\omega_{i_{k}}(\alpha)$. As for the difference of any two connections we have

$$
i\left(x^{*}\right)\left(\omega_{i_{j}}(\alpha)-\omega_{i_{k}}(\alpha)\right)=\alpha(x)-\alpha(x)=0 \text { for } x \in \mathfrak{g},
$$

where $x^{*}$ denotes the canonical vertical vector field on $P$ determined by $x \in g$. Since the connections $\omega_{i_{j}}$ and $\omega_{i_{k}}$ are adapted, they annihilate the foliation $\widetilde{L} \subset T_{P}$ (projecting onto $L \subset T_{M}$ ). It follows that the 1forms $\omega_{i_{j}}(\alpha)-\omega_{i_{k}}(\alpha)$ on $\pi^{-1}\left(U_{\left(i_{j}, i_{k}\right)}\right)$ are locally representable as $\sum_{l} \pi^{*} \gamma_{l} \cdot f_{l}$, where $\gamma_{l} \in Q^{*}$ and $f_{l} \in \Omega_{P}^{*}$. This proves (6.5).

To prove (6.6) we observe that $k\left(\omega_{o}\right) \widetilde{\beta}^{(j)}=k\left(\omega_{i_{j}}\right) \widetilde{\beta}$, i.e., $k\left(\omega_{o}\right) \widetilde{\beta}^{(j)}$ is a curvature term of the adapted connection $\omega_{i_{j}}$ on $P / U_{i_{j}}$. But, as for the curvature of any connection, we have then $i\left(x^{*}\right) k\left(\omega_{i_{j}}\right) \widetilde{\beta}=0, x \in \mathfrak{g}$. 
Since $\omega_{i j}$ is adapted and $\tilde{L}$ is involutive it follows that

$$
k\left(\omega_{i_{j}}\right) \widetilde{\beta}(X, Y)=d \omega(\beta)(X, Y)+\beta[\omega(X), \omega(Y)]=0,
$$

for germs of vector fields $X, Y$ in $\widetilde{L}$. It follows that the 2 -forms $k\left(\omega_{i_{j}}\right) \widetilde{\beta}$ on $\pi^{-1}\left(U_{i_{j}}\right)$ are locally representable as $\sum_{l} \pi^{*} \gamma_{l} \wedge \gamma_{l}^{\prime}$, where $\gamma_{l} \in \mathscr{Q}^{*}$ and $\gamma_{l}^{\prime} \in \Omega_{P}^{1}$. This proves (6.6).

Since by (6.3) we have $F^{q+1} \breve{C}\left(\mathscr{C}, \pi_{*} \Omega_{P}^{*}\right)=0$, it follows that $k_{1}(\omega)$ induces a $\mathfrak{g}$-DG-algebra homomorphism (still denoted $k_{1}(\omega)$ ) of the form

$$
k_{1}(\boldsymbol{\omega}): W_{1}(\mathfrak{g})_{q} \equiv W_{1}(\mathfrak{g}) / F_{1}^{2(q+1)}(\mathfrak{g}) W_{1}(\mathfrak{g}) \rightarrow \breve{C}\left(\mathscr{C}, \pi_{*} \Omega_{P}^{*}\right) .
$$

Let now $H \subset G$ be a closed subgroup with finitely many connected components (taken in the appropriate category). By passage to $H$-basic elements we obtain then a DG-algebra homomorphism

$$
k_{1}(\omega): W_{1}(\mathfrak{g}, H)_{q} \rightarrow \check{C}\left(\mathscr{K}, \hat{\pi}_{*} \Omega_{P / H}\right) .
$$

Here $\hat{\pi}: P / H \rightarrow M$ denotes the projection induced by $\pi: P \rightarrow M$. Let $s$ : $M \rightarrow P / H$ be a cross-section of $\hat{\pi}$, defining an $H$-reduction of the $G$-bundle $P$. By composition we obtain then a homomorphism of DG-algebras

$$
\Delta(\omega)=s^{*} \circ k_{1}(\omega) \circ \lambda
$$

which completes the following diagram

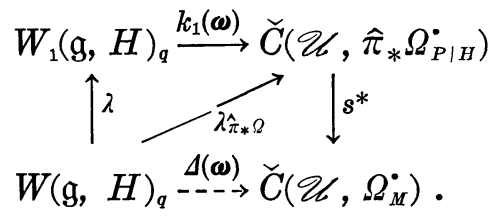

Recall that by Theorem 5.21 and Remark 5.22 the map $\lambda$ induces an algebra-isomorphism in cohomology and thus $k_{1}(\omega)$ and $\lambda_{\hat{\pi}_{*} \Omega}$ determine the same multiplicative maps in cohomology. The following argument shows that in fact the cohomology maps $k_{1}(\omega)_{*}$ and $\Delta(\omega)_{*}$ are independent of the family of local adapted connections on $\mathscr{U}$. Given two families of local adapted connections $\omega_{0}$ and $\omega_{1}$ on $\mathscr{U}$, we observe that by (3.33) we have a homomorphism of $\mathfrak{g}$-DG-algebras

$$
k_{2}\left(\omega_{0}, \omega_{1}\right): W_{2}(\mathfrak{g}) \rightarrow C\left(\Delta_{(1)}, \check{C}\left(\mathscr{U}, \pi_{*} \Omega_{P}^{*}\right)\right),
$$

where $\Delta_{(1)}$ is the standard ss set with two vertices. As in Proposition 6.4 it follows that $k_{2}\left(\omega_{0}, \omega_{1}\right)$ preserves filtrations and therefore we obtain a commutative diagram analogous to (3.39) 


$$
\begin{gathered}
H^{*}\left(W_{2}(\mathfrak{g}, H)_{q}\right) \stackrel{k_{2}\left(\omega_{0}, \omega_{1}\right)_{*}}{\longrightarrow} H^{*}\left(C\left(\Delta_{(1)}, \breve{C}\left(\mathscr{U}, \hat{\pi}_{*} \Omega_{P / H}\right)\right)\right) \\
\cong \unrhd\left(\rho_{2}\right)_{*} \\
\cong \backslash\left(j_{l}\right)_{*} \\
H^{*}\left(W_{1}(\mathfrak{g}, H)_{q}\right) \stackrel{k_{1}\left(\omega_{l}\right)_{*}}{\longrightarrow} H^{*}\left(C\left(\mathscr{U}, \hat{\pi}_{*} \Omega_{P / H}^{\cdot}\right)\right) .
\end{gathered}
$$

By Proposition 3.38, Theorem 5.5 and Remark 5.22 the vertical maps are isomorphisms which are independent of $l=0,1$. It follows that $k_{1}\left(\omega_{l}\right)_{*}=\left(j_{l}\right)_{*} \circ k_{2}\left(\omega_{0}, \omega_{1}\right)_{*} \circ\left(\rho_{2}\right)_{*}^{-1}=\varepsilon_{*}^{-1} \circ k_{2}\left(\omega_{0}, \omega_{1}\right)_{*} \circ\left(\rho_{2}\right)_{*}^{-1}$ is independent of $l=$ 0,1 . Hence we have proved the following result.

PRoposition 6.11. The homomorphisms of graded algebras

$$
k_{1}(\omega)_{*}: H^{*}\left(W_{1}(\mathfrak{g}, H)_{q}\right) \rightarrow H^{*}\left(\mathscr{U} ; \hat{\pi}_{*} \Omega_{P \mid H}^{\cdot}\right)
$$

and

$$
\Delta(\omega)_{*}: H^{*}\left(W(\mathrm{~g}, H)_{q}\right) \rightarrow H^{*}\left(\mathscr{Q} ; \Omega_{M}^{*}\right)
$$

are independent of the choice of the family of adapted connection $\omega=$ $\left(\omega_{j}\right)$ on $P / \mathscr{U}$.

From now on we will denote these two cohomology maps by

$$
\begin{gathered}
k_{1}(P, \mathscr{C})_{*}: H^{*}\left(W_{1}(\mathfrak{g}, H)_{q}\right) \rightarrow H^{*}\left(\mathscr{U} ; \hat{\pi}_{*}\left(\Omega_{P \mid H}^{\cdot}\right)\right) \\
\Delta(P, \mathscr{U})_{*}=s^{*} k_{k_{1}}(P, \mathscr{U})_{*} \circ \lambda_{*}: H^{*}\left(W(\mathfrak{g}, H)_{q}\right) \rightarrow H^{*}\left(\mathscr{U} ; \Omega_{M}^{*}\right) .
\end{gathered}
$$

These maps depend only on the foliated bundle $P \stackrel{\pi}{\rightarrow} M$ and, in the case of $\Delta(P, \mathscr{C})_{*}$, also on the $H$-reduction $s$ of $P$.

As an immediate consequence of Proposition 6.11 we get the compatibility of the maps $\Delta(P, \mathscr{C})_{*}$ under refinements of $\mathscr{C}$. If indeed $\mathscr{V}=\left(V_{i}\right)_{i \in I}$ is a refinement of $\mathscr{Q} b$ via an index $\operatorname{map} \alpha: I \rightarrow J$ and if $\omega=\left(\omega_{j}\right)_{j \in J}$, resp. $\omega^{\prime}=\left(\omega_{i}^{\prime}\right)_{i \in I}$ are families of adapted connections on $P / \mathscr{U}$, resp. $P / \mathscr{V}$, then $\alpha^{*}(\omega)=\left(\omega_{\alpha(i)} \mid V_{i}\right)_{i \in I}$ is a second family of adapted connections on $P / \mathscr{V}$. From Proposition 6.11 we get the commutative diagrams

$$
\begin{aligned}
& H^{\cdot}\left(\mathscr{U} ; \pi_{*} \Omega_{P \mid H}^{\cdot}\right) \stackrel{j_{\alpha_{*}}}{\longrightarrow} H^{*}\left(\mathscr{V} ; \pi_{*} \Omega_{P \mid H}^{\cdot}\right), \\
& k_{1}(P, \mathscr{U})_{*} \backslash \overrightarrow{k_{1}(P, \mathscr{V})_{*}} \\
& H\left(W_{1}(\mathfrak{g}, H)_{q}\right)
\end{aligned}
$$

and

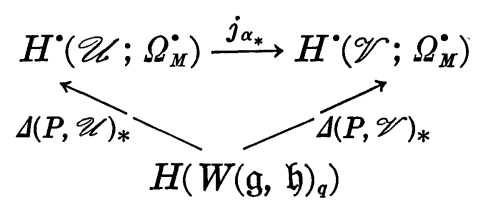


where $j_{\alpha_{*}}$ denotes the canonical map induced by $\alpha$. So far the target of the map $\Delta(P, \mathscr{U})_{*}$ is the cohomology algebra $H\left(\mathscr{U} ; \Omega_{M}^{*}\right)$ and therefore depends a priori on the covering $\mathscr{U}$. The passage to the De Rham cohomology of $M$ can now be done by standard methods of sheaf-cohomology theory [7, Ch. II, Sections 5.3 and 5.4], [8]. To allow the interpretation of the general theory in various categories, we view the De Rham cohomology of $M$ as the hypercohomology $H\left(M ; \Omega_{M}^{*}\right)$ of $M$ with coefficients in the sheaf complex $\Omega_{M H}^{*}$ of differential forms (with respect to the structure sheaf $\left.\mathcal{O}_{M}\right)$ on $M$, i.e., $H_{\mathrm{D}_{\mathrm{R}}}^{\cdot}(M) \equiv \boldsymbol{H}^{*}\left(M ; \Omega_{M}^{*}\right)=H^{*}\left(\Gamma\left(M ; \mathscr{C}\left(M, \Omega_{M}^{*}\right)\right)\right)$ where $\mathscr{C}(M,-)$ is the canonical resolution on $M$ [8], [9]. From the triple-complex

$$
K=\check{C}^{\bullet}\left(\mathscr{U}, \mathscr{C}^{\bullet}\left(M, \Omega_{M}^{*}\right)\right)
$$

we obtain then the following information. Filtering $K$ with respect to degrees $\geqq p$ in $\mathscr{C} \cdot(M,-)$ we get a spectral sequence whose $E_{1}$-term is given by

$$
" E_{1}^{r, s}=H^{s}\left(\mathscr{C} ; \mathscr{C}^{r}\left(M, \Omega_{M}^{*}\right)\right) .
$$

Thus $" E_{1}^{r, s}=0$ for $s>0$, since $\mathscr{C}^{r}(M,-)$ is flabby, and we have an edge isomorphism

$$
j_{*}^{\prime \prime}: \boldsymbol{H}\left(M ; \Omega_{M}\right)=H_{\mathrm{DR}}(M) \stackrel{\cong}{\longrightarrow} H\left(K^{t \circ t}\right)
$$

induced by the canonical inclusion $j^{\prime \prime}: \mathscr{C}\left(M, \Omega_{M}^{*}\right) \rightarrow K$. The first spectral sequence of $K$ (with respect to the complementary degree) is then of the form

$$
{ }^{\prime} E_{2}^{r, s} \cong H^{r}\left(\mathscr{U} ; \underset{\mathscr{H}^{s}}{ }\left(\Omega_{M}^{\cdot}\right)\right) \Rightarrow H_{\mathrm{DR}}^{r+s}(M),
$$

where $\mathscr{H}^{s}\left(\Omega_{M}^{*}\right)$ is the local system of coefficients on $N(\mathscr{U})$ given by $\sigma \mapsto H^{s}\left(\widetilde{U}_{o}, \Omega_{M}^{*}\right)=H^{s}\left(\Gamma\left(U_{o}, \mathscr{C}\left(M, \Omega_{M}^{*}\right)\right),\right) s \geqq 0$. Thus we see that the edge maps

$$
\left(j_{\mathscr{C}}\right)_{*}: H^{n}\left(\mathscr{C} ; \Omega_{M}^{*}\right) \stackrel{j_{*}^{\prime}}{\longrightarrow} H^{n}\left(K^{t o t}\right) \stackrel{j_{*}^{\prime \prime}}{\cong} H_{\mathrm{DR}}^{n}(M)
$$

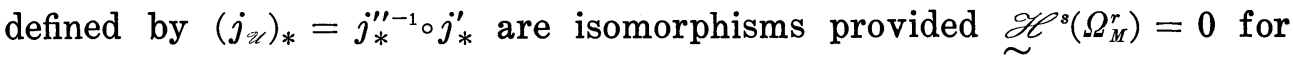
$s>0$, i.e., if the sheaves $\Omega_{M}^{r}$ are $\Gamma\left(U_{o},-\right)$-acyclic. If the sheaves $\Omega_{m}^{r}$ are in addition globally acyclic, i.e., $\Gamma(M,-)$-acyclic, then by a similar argument the canonical inclusion

$$
i_{\varkappa}: \Gamma\left(M, \Omega_{M}^{*}\right) \rightarrow \breve{C}\left(\mathscr{U} ; \Omega_{M}^{*}\right)
$$

into $\breve{C}^{0}$ induces an isomorphism in cohomology (compare Remarks 6.23, 6.24 , and 6.25). 
The composition

$$
\Delta(P)_{*}=\left(j_{\mathscr{U}}\right)_{*} \circ \Delta(P, \mathscr{U})_{*}
$$

is called the semi-simplicial characteristic homomorphism of the foliated bundle $P \stackrel{\pi}{\rightarrow} M$ relative to the $H$-structure $\bar{s}: P^{\prime} \subset P$ defined by $s: M \rightarrow$ $P / H$. It has been introduced and applied in [13], [14], [15]. The map $\Delta(P)_{*}$ depends only on the foliated structure of $P$ and the $H$-reduction $s$ (see Remark 6.23 for a case where $\Delta(P)_{*}$ is even independent of $s$ ). In addition to the primary characteristic classes of $P$ satisfying a Bott vanishing theorem, $\Delta(P)_{*}$ defines characteristic classes of secondary type, which are obstructions to the invariance of the $H$-subbundle $P^{\prime} \subset_{\bar{s}} P$ under the flows generated by vector fields tangent to the foliation on $P$ (compare [14], [16], and [17], [18] for explicit calculations and applications).

Furthermore, if the foliated bundle $P \stackrel{\pi}{\rightarrow} M$ admits a global adapted connection $\omega: \wedge^{*} \mathrm{~g} \rightarrow \Omega(P)$, we get from Proposition 3.41 a commutative diagram derived from (3.43)

$$
\begin{array}{cc}
\Gamma\left(M, \Omega_{M}^{*}\right) \stackrel{i »}{\longrightarrow} \breve{C}\left(\mathscr{U} ; \Omega_{M}^{*}\right) \\
\uparrow s^{* \circ k(\omega)} & \uparrow \Delta(\omega) \\
W(\mathrm{~g}, H)_{q} \stackrel{\lambda^{\prime}}{\longrightarrow} W_{1}(\mathrm{~g}, H)_{q},
\end{array}
$$

where $s^{*} \circ k(\omega): W(\mathfrak{g}, H)_{q} \stackrel{k(\omega)}{\longrightarrow} \Gamma\left(P / H, \Omega_{P \mid H}^{*}\right) \stackrel{s^{*}}{\longrightarrow} \Gamma\left(M, \Omega_{M}^{*}\right)$ is the composition of the ordinary Weil homomorphism $k(\omega)$ of $\omega$ with the map $s$ defining the $H$-reduction of $P$. Thus in the presence of a global adapted connection the direct construction of the generalized characteristic homomorphism via $s^{*} \circ k(\omega)$ (see [16], Theorem 4.43 ) gives the same result as the ss construction even at the cochain level. We summarize the construction of the ss characteristic homomorphism $\Delta(P)_{*}$ as follows.

THEOREM 6.21. Let $\pi: P \rightarrow M$ be a foliated G-bundle with $G$ connected and $q$ the codimension of the foliation on the base space $M$. Let $H \subset G$ be a closed subgroup with finitely many connected components, and an $H$-reduction of $P$ given by a cross-section $s: M \rightarrow P / H$ of the associated bundle $\hat{\pi}: P / H \rightarrow M$. Then the map $\Delta(P)_{*}$ in the commutative diagram

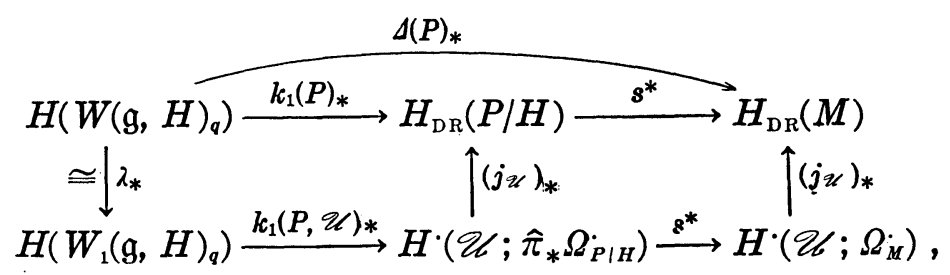


is an algebra homomorphism depending only on the foliated bundle $P$ and the $H$-reduction $s$ of $P$. It is determined on $H\left(W_{1}(\mathfrak{g}, H)_{q}\right)$ by a family $\omega=\left(\omega_{j}\right)$ of adapted connections on $P / \mathscr{C} . \Delta(P)_{*}$ is functorial in all data. Since $\lambda_{*}$ is an algebra-isomorphism, this implies that $H \cdot\left(W(\mathfrak{g}, H)_{q}\right)$ and $H \cdot\left(W_{1}(\mathfrak{g}, H)_{q}\right)$ can be used interchangeably as domain of definition for $\Delta(P)_{*}$. We have further the following properties.

(i) If the open covering $\mathscr{U}=\left(U_{j}\right)_{j \in \Delta}$ satisfies the condition

$$
H^{s}\left(U_{o}, \Omega_{M}^{r} / U_{o}\right)=0, \text { for } s>0, \quad r \geqq 0
$$

for every $\sigma \in N(\mathscr{U})$, then the target of $\Delta(P)_{*}$ is given by

$$
H \cdot\left(\mathscr{C} ; \Omega_{M}^{\cdot}\right) \underset{\left(j_{\mathscr{C}}\right) *}{\cong} H_{\mathrm{DR}}^{\cdot}(M),
$$

and $\Delta(P)_{*}$ can be realized by the map $\Delta(P, \mathscr{C})_{*}$ into $H\left(\mathscr{U} ; \Omega_{M}^{*}\right)$, the total cohomology of the double complex $\breve{C}\left(\mathscr{U}, \Omega_{M}^{*}\right)$.

(ii) Assume in addition that the foliated bundle $P$ admits a global adapted connection $\omega$ and that $H^{s}\left(M, \Omega_{M}^{r}\right)=0$ for $s>0$ and $r \geqq 0$. Then the target of $\Delta(P)_{*}$ is given by

$$
H^{\cdot}\left(\Gamma\left(M, \Omega_{M}^{\cdot}\right)\right) \underset{(i \overline{z \mathcal{K}})_{*}}{\cong} H^{\cdot}\left(\mathscr{U} ; \Omega_{M}^{\cdot}\right) \underset{(j \overline{\mathcal{K}})_{*}}{\cong} H_{\mathrm{DR}}^{\cdot}(M)
$$

and $\Delta(P)_{*}$ can be realized by the map $s^{*} \circ k(\omega)_{*}$ into the cohomology of global forms on $M$.

We conclude with a few remarks about specific situations in which this general theory applies.

Sмоoтн CASE 6.23. If all structures involved are smooth and $M$ is paracompact, the modules $\Omega_{M}^{p}$ and $\Omega_{M}^{p} / U_{o}$ are fine. Therefore the maps in (6.17), (6.18) induce isomorphisms in cohomology. Using a partition of unity one obtains also a global adapted connection $\omega$ on $P$. Hence part (ii) of Theorem 6.21 applies. If further $H=K_{G}$ is a maximal compact subgroup of $G$, there exists (up to homotopy), a unique $K_{G}$-reduction of $P$ and therefore $\Delta(P)_{*}$ depends on $P$ only. If $P$ is in particular the canonically foliated frame bundle of a codimension $q$ foliation on $M$ given in terms of a Haefliger $\Gamma_{q}$-cocycle, the semi-simplicial construction given here defines the characteristic homomorphism directly in terms of that cocycle. In this case our construction coincides with the characteristic homomorphism of foliations in Bott-Haefliger [3] [10]; compare also Bott-Shulman-Stasheff [4], Shulman [24], Shulman-Stasheff [25] and Vey [26].

CompleX-ANALYTIC CASE 6.24. In this situation the appropriate coverings $\mathscr{C}$ of $M$ are Stein coverings, where the $U_{\sigma} \subset M$ are Stein-manifolds for 
$\sigma \in N(\mathscr{C})$. By Cartan's Theorem A the cohomology groups $H^{s}\left(U_{o}, \Omega_{M}^{r} / U_{o}\right)$ are zero for $s>0$, and part (i) of Theorem 6.21 applies with respect to families of holomorphic adapted connections. Some explicit calculations in this context can be found in [15]. In this context the subgroup $H$ entering into Theorem 6.21 need not necessarily be complex-analytic. One simply passes then to the smooth De Rham complex $\mathscr{A}_{C}(M)$ on the RHS of diagram (6.22), prior to applying the map $s^{*}$.

Algebraic CASE 6.25. In the category of algebraic varieties (or schemes) over a ground field of characteristic zero our construction applies in much the same way as in 6.24. In particular Stein coverings will have to be replaced by affine coverings. Even in the absence of foliations one obtains in this way a semi-simplicial description of the characteristic homomorphism

$$
\Delta_{*}: I(G) \stackrel{\lambda_{*}}{\longrightarrow} H\left(W_{1}(\mathfrak{g}, G)\right) \stackrel{k_{1}(P, \mathscr{U})}{\longrightarrow} H\left(\mathscr{U}, \Omega_{M}^{\cdot}\right) \cong H_{\mathrm{DR}}(M)
$$

of an algebraic G-bundle (compare Illusie [11]).

CoHomology of $B G$ 6.26. We finally observe that our theory yields a semi-simplicial realization of the Chern-Weil homomorphism for ordinary G-bundles $P$ using local connection data in $P$ [13] [14]. In particular we obtain for the universal $G$-bundle $E G \rightarrow B G$ an isomorphism

$$
\Delta_{*}: I\left(K_{G}\right) \cong H\left(W_{1}\left(\mathfrak{g}, K_{G}\right)\right) \stackrel{\cong}{\longrightarrow} H(B G, \boldsymbol{R}),
$$

and a spectral sequence

$$
E_{2}^{2 p, q} \cong H^{q}\left(\mathfrak{g}, K_{G}\right) \otimes I(G)^{2 p} \Rightarrow H\left(W_{1}\left(\mathfrak{g}, K_{G}\right)\right),
$$

whose edge-homomorphism is the universal Chern-Weil homomorphism

$$
I(G) \rightarrow H\left(W_{1}\left(\mathfrak{g}, K_{G}\right)\right) \cong H(B G, \boldsymbol{R})
$$

(compare [15], 2.11). In this case our construction coincides with the results in Bott [2] via the van Est theorem.

\section{REFERENCES}

[1] R. Botr, Lectures on characteristic classes and foliations, Springer Lecture Notes 279 (1972), 1-94.

[2] R. Bотт, On the Chern-Weil homomorphism and the continuous cohomology of Lie groups, Advances in Math. 11 (1973), 289-303.

[3] R. BotT AND A. Hakfliger, On characteristic classes of $\Gamma$-foliations, Bull. Amer. Math. Soc. 78 (1972), 1039-1044.

[4] R. Bott, H. Shulman, and J. StashefF, On the De Rham theory of certain classifying spaces, Advances in Math. 20 (1976), 43-56. 
[5] H. Cartan, Cohomologie réelle d'un espace fibré principal différentiable, Séminaire Cartan, exposés 19 et $20(1949 / 50)$.

[6] C. Godbillon and J. Vey, Un invariant des feuilletages de codimension un, C. R. Ac. Sci. Paris, t. 273 (1971), 92-95.

[7] R. Godement, Topologie algébrique et théorie des faisceaux, Hermann, Paris (1958).

[8] A. Grothendieck, Sur quelques points d'algèbre homologique, Tohôku Math. J. 9 (1957), 119-221.

[9] A. Grothendieck, On the De Rham cohomology of algebraic varieties, Publ. Math. IHES 29 (1966), 95-103.

[10] A. HAEFLIGER, Sur les classes caractéristiques des feuilletages, Séminaire Bourbaki, $24 \mathrm{e}$ année $1971 / 72, \mathrm{n}^{\circ} 412$.

[11] L. ILLUSIE, Complexe cotangent et déformations, I et II, Springer Lecture Notes 239 (1971) and 283 (1972).

[12] F. Kamber and Ph. Tondeur, Cohomologie des algèbres de Weil relatives tronquées, C. R. Acad. Sci. Paris, t. 276 (1973), 459-462.

[13] F. Kamber and Ph. Tondeur, Algèbres de Weil semi-simpliciales, C. R. Ac. Sci. Paris, t. 276 (1973), 1177-1179; Homomorphisme caractéristique d'un fibré principal feuilleté, ibid, 1407-1410; Classes caractéristiques dérivées d'un fibré principal feuilleté, ibid, $1449-1452$.

[14] F. Kamber and Ph. Tondeur, Characteristic invariants of foliated bundles, Manuscripta Mathematica 11 (1974), 51-89.

[15] F. Kamber and Ph. Tondeur, Semi-simplicial Weil algebras and characteristic classes for foliated bundles in Čech cohomology, Proc. Symposia Pure Math. Stanford 1973, Vol. 27 (1975), Part I, 283-294.

[16] F. Kamber and Ph. TondeUR, Foliated bundles and characteristic classes, Springer Lecture Notes 493 (1975), 1-208.

[17] F. Kamber and Ph. Tondeur, Non-trivial characteristic invariants of homogeneous foliated bundles, Ann. Scient. Ec. Norm. Sup. 8(1975), 433-486.

[18] F. Kamber and PH. Tondeur, On the linear independence of certain cohomology classes of $B \Gamma$, Advances in Math., to appear.

[19] F. Kamber and Ph. TondeUr, Characteristic classes of complex analytic foliated bundles, to appear.

[20] F. Kamber and Ph. Tondeur, Cohomology of g-DG-algebras, to appear.

[21] J. L. Koszul, Multiplicateurs et classes caractéristiques, Trans. Amer. Math. Soc. 89 (1958), 256-266.

[22] J. L. Koszul, Espaces fibrés et pré-associés, Nagoya Math. J. 15 (1959), 155-169.

[23] G. SegAL, Classifying spaces and spectral sequences, Publ. Math. IHES 34 (1968), 105112.

[24] H. Shulman, Characteristic classes of foliations, Ph. D. Thesis, University of California, Berkeley (1972).

[25] H. Shulman and J. StashefF, De Rham theory for $B \Gamma$, Springer Lecture Notes 652 (1978), 62-74.

[26] J. VeY, Quelques constructions relatives aux $\Gamma$-structures, C. R. Acad. Sc. Paris, t. 276 (1973), 1151-1153.

[27] A. Weil, Sur les théorèms de De Rham, Comment. Math. Helv. 26 (1952), 119-145.

UNIVERSITY OF ILLINOIS

at URBana-Champaign

U.S.A. 